\title{
Review
}

\section{Itinerant ferromagnetism in narrow-band metals}

\author{
P. Farkašovský \\ Institute of Experimental Physics, Slovak Academy of Sciences, \\ Watsonova 47, 04353 Košice, Slovakia
}

Received August 11, 2021, in final form October 19, 2021

Since its introduction in 1963, the Hubbard model has becomes one of the most popular models used in the literature to study cooperative phenomena in narrow-band metals (ferromagnetism, metal-insulator transitions, charge-density waves, high-T $c$ superconductivity). Amongst all these cooperative phenomena, the problem of itinerant ferromagnetism in the Hubbard model has the longest history. However, in spite of an impressive research activity in the past, the underlying physics (microscopic mechanisms) that leads to the stabilization of itinerant ferromagnetism in Hubbard model (narrow-band metals) is still far from being understood. In this review we present our numerical results concerning this subject, which have been reached by small cluster exact diagonalization, density matrix renormalization group and quantum Monte Carlo calculations within various extensions of the Hubbard model. Particular attention is paid to a description of crucial mechanisms (interactions) that support the stabilization of the ferromagnetic state, and namely: (i) the long-range hopping, (ii) the correlated hopping, (iii) the long-range Coulomb interaction, (iv) the flat bands and (v) the lattice structure. Most of the presented results have been obtained for the one-dimensional case, but the influence of the increasing dimension of the system on the ferromagnetic state is also intensively discussed.

Key words: itinerant ferromagnetism, correlated electron systems, Hubbard model

\section{Introduction}

The microscopic description of itinerant ferromagnetism in narrow-band metals is one of the most interesting as well as the most complicated many-particle problems in condensed matter physics. This is expected to be due to the interplay between ordinary, spin-independent Coulomb interaction (strong and strongly screened) and kinetic energy of itinerant electrons within the frames determined by the Pauli exclusion principle. The single-band Hubbard model [1], possibly the simplest lattice model of correlated electrons, was first thought to encompass a minimal description of band-ferromagnetism. The Hamiltonian of this model can be written as a sum of two terms:

$$
H=\sum_{i j \sigma} t_{i j} c_{i \sigma}^{+} c_{j \sigma}+U \sum_{i} n_{i \uparrow} n_{i \downarrow}
$$

where $c_{i \sigma}^{+}$and $c_{i \sigma}$ are the creation and annihilation operators for an electron of spin $\sigma=\uparrow, \downarrow$ at site $i$ and $n_{i \sigma}$ is the corresponding number operator, which counts the number of electrons of spin $\sigma$ on site $i$.

The first term of (1.1) is the kinetic energy of itinerant electrons. It corresponds to quantum-mechanical hopping of the $\sigma$-spin electrons between sites $i$ and $j$, with transition hopping probabilities $t_{i j}$. Usually, it is assumed (the ordinary Hubbard model) that $t_{i j}=-t$ if $i$ and $j$ are nearest neighbors and $t_{i j}=0$ otherwise.

The second term corresponds to the Coulomb repulsion between two electrons of opposite spins at the same site. The long-range contribution is assumed to be screened and only the interaction when both the $\sigma=\uparrow$ and $\sigma=\downarrow$ electrons are on the same atom is retained, yielding an additional energy of $U$ when the atom is doubly occupied. 
Thus, naively the ordinary Hubbard model might be thought to describe the competition between the kinetic energy and the short-range Coulomb interaction, but in fact there is the third "force" at work which severely complicates the problem: Pauli exclusion. Electrons are fermions and so the many-particle wave function must be antisymmetric under interchange of any two electrons. Unlike the first two forces which are basically short-range interactions, antisymmetrizing wave functions are effectively a long-range interaction that significantly complicates a description of correlation effects within, at the first glance, the very simple model. On the other hand, there were very pleasurable arguments which, at least in the first stages of the study, favored the Hubbard model as the generic model for a description of the band ferrommagnetism in the transition metal compounds. Indeed, writing the magnetization as $m_{i}=n_{i \uparrow}-n_{i \downarrow}$ and total number of $n$ electrons (per site) as $n_{i}=n_{i \uparrow}+n_{i \downarrow}$, the interaction part of the Hubbard model can be rewritten as $U n_{i \uparrow} n_{i \downarrow}=U\left(n_{i}^{2}-m_{i}^{2}\right) / 4$. Since the number $n$ of electron per atom is fixed, the Coulomb interaction favors the formation of a magnetic moment.

However, the subsequent studies of the model showed that the single-band Hubbard model is not the canonical model for ferromagnetism. Indeed, the existence of saturated ferromagnetism has been proven rigorously only for very special limits. The first well-known example is the Nagaoka ferromagnetism that comes from the Hubbard model in the limit of infinite repulsion and one hole in a half-filled band [2]. Another example, where saturated ferromagnetism has been shown to exist, is the case of the one-dimensional Hubbard model with nearest and next-nearest-neighbor hopping at low electron densities [3]. Furthermore, several examples of the fully polarized ground state have been found on special lattices as are the bipartite lattices with sublattices containing a different number of sites [4], the fcc-type lattices [5, 6], the lattices with long-range electron hopping [7, -9], the flat bands [10-14] and the nearly flat-band systems [15-23]. This indicates that the lattice structure, which dictates the shape of the density of states (DOS), plays an important role in stabilizing the ferromagnetic state.

To examine in more detail the role of these (and some other) factors on the stabilization of the ferromagnetic state in the generalized Hubbard model, in our previous studies we have used smallcluster-exact diagonalization [24], density matrix renormalization group [25, 27] and projector-quantum Monte Carlo[28-30] calculations. In particular, we have examined the role of the long-range hopping [31, 32] and long-range Coulomb interaction [33] with exponentially decaying amplitudes, the correlated hopping [34], the flat bands [35] and the lattice structure [36, 37]. The main results of our numerical studies are summarized in this short review.

\section{Results and discussion}

\subsection{The effect of long-range hopping}

\subsubsection{One-dimensional case}

Since the model including the electron hopping only to the nearest neighbors may seem at first glance a very crude approximation, in order to have a more realistic description of electron processes in transition metal compounds, we have generalized this model by taking into account also transitions to next neighbors. Basically, there are two possible ways of performing such a generalization. The first way is to assign independent transition amplitudes for the first $\left(t_{1}\right)$, second $\left(t_{2}\right)$, third $\left(t_{3}\right)$, forth $\left(t_{4}\right), \ldots$ nearest neighbors, while the second way is to describe the electron hopping by a simple one-parametric formula [38, 39] with exponentially decaying hopping amplitudes between $\mathbf{R}_{\mathbf{i}}$ and $\mathbf{R}_{\mathbf{j}}$ lattice sites, i.e.,

$$
t_{i, j}(q)=\left\{\begin{array}{cc}
0, & \mathbf{R}_{\mathbf{i}}=\mathbf{R}_{\mathbf{j}} \\
-q^{\left|\mathbf{R}_{\mathbf{i}}-\mathbf{R}_{\mathbf{j}}\right|} / q, & \mathbf{R}_{\mathbf{i}} \neq \mathbf{R}_{\mathbf{j}}
\end{array}\right.
$$

where $q$ is the parameter that controls the range of electron hopping $(0 \leqslant q \leqslant 1)$. From the practical point of view, the second method is more suitable because it does not expand the model parameter space and has a clearer physical meaning, since the atomic wave functions have also the exponential decay with increasing distance. For this reason, for a description of electron hopping in the generalized model, we have chosen the long-range hopping with exponentially decreasing amplitudes. 


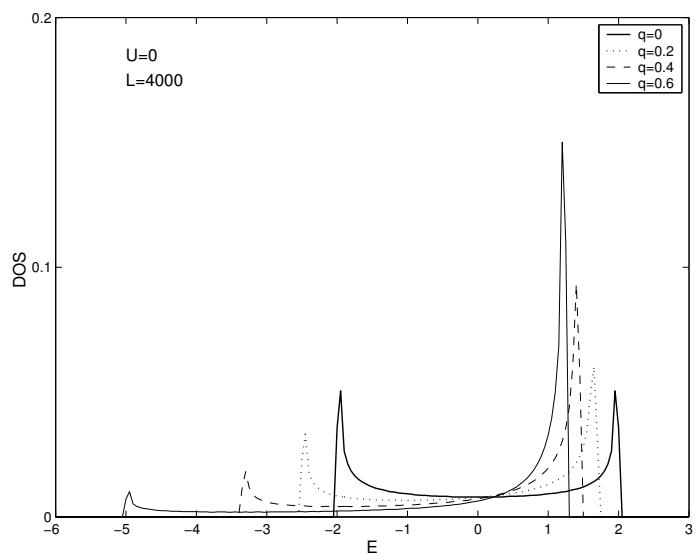

Figure 1. The non-interacting ( $D=1)$ DOS corresponding to the long-range hopping for different $q$ and $L=4000[31]$.

The selection of hopping matrix elements in the form given by equation (2.1) has several advantages. It represents a much more realistic type of electron hopping on a lattice (in comparison to nearest-neighbor hopping), and it allows us to change continuously the type of hopping (band) from nearest-neighbor $(q=0)$ to infinite-range $(q=1)$ hopping and thus immediately study the effect of the long-range hopping. Another advantage follows from figure 1. where the density of states (DOS) corresponding to equation (2.1) is displayed for several values of $q$. It is seen that with increasing $q$, more weight shifts to the upper edge of the band and the DOS becomes strongly asymmetric. Thus, one can simultaneously study (by changing only one parameter $q$ ) the influence of the increasing asymmetry in the DOS and the influence of the long-range hopping on the ground state properties of the Hubbard model.

The Hamiltonian of the single-band Hubbard model with long-range hopping is given by:

$$
H=\sum_{i j \sigma} t_{i j}(q) c_{i \sigma}^{+} c_{j \sigma}+U \sum_{i} n_{i \uparrow} n_{i \downarrow} .
$$

The exact results on the ground states of the Hubbard model with the generalized type of hopping (2.2) exist only for the special case of $q=1$ when the electrons can hop to all sites with equal probabilities [7. 9]. For this type of hopping and the electron filling just above half-filling ( $N=\sum_{\sigma} N_{\sigma}=L+1$, where $L$ is the number of lattice sites), it was shown that the ground state is not degenerate with respect to the total spin $S$ and it is maximum ferromagnetic with $S=(L-1) / 2$ (for all $U>0$ ). For higher fillings $(N>L+1)$, the ferromagnetic ground state still exists but it is completely degenerate with respect to $S$. The limit of infinite-range hopping is, however, the least realistic limit of equation (2.1). It is interesting, therefore, to look at the possibility of ferromagnetism in the Hubbard model with a generalized type of hopping for smaller values of $q$ that describe a much more realistic type of electron hopping.

In our paper [31] we have extended calculations to arbitrary $q$ and arbitrary band fillings $n=N / L$. The ground states of the model have been determined by exact diagonalizations for a wide range of model parameters $(q, U, n)$. Typical examples are chosen from a large number of available results to represent the most interesting cases. The results obtained are presented in the form of phase diagrams in the $U-q$ plane. To determine the phase diagram in the $U-q$ plane (corresponding to some $L$ and $n$ ), the ground state energy of the model is calculated point by point as functions of $q$ and $U$. Of course, such a procedure demands in practice a considerable amount of CPU time, which imposes severe restrictions upon the size of clusters that can be studied using this method $(L \sim 16)$. Fortunately, we have found that the ground-state energy of the model depends on $L$ only very weakly (for a wide range of the model parameters) and thus already such small clusters can satisfactorily describe the ground state properties of the model.

Although the appearance of the ferromagnetic state at $q=1$ and $N=L+1$ (discussed above) is interesting from the theoretical point of view, in the thermodynamic limit $(L \rightarrow \infty)$ this result is not 
significant if the ferromagnetic state does not persist also for higher fillings. Analytical results obtained for $q=1$ predict, however, that the ground states for $N>L+1$ are completely degenerate with respect to the total spin $S$ and thus the only possibility for the stabilization of the ferromagnetic state is that the long-range hopping with $q \neq 1$ removes this degeneracy. Numerical calculations that we have performed for a wide range of electron fillings $n>1$ fully confirmed this assumption. It was found that the long-range hopping with $q \neq 1$ not only removes the degeneracy of the ground states with respect to $S$ but at the same time stabilizes the ferromagnetic state. Furthermore, these calculations showed that the effect of the long-range hopping on the stability of the ferromagnetic state is extremely strong, especially for small values of $q$. The results of our small-cluster exact-diagonalization calculations obtained on finite clusters up to $L=16$ sites are summarized in figure 2. There is shown a critical interaction strength $U_{c}$, above which the ground state is ferromagnetic, as a function of $q$ for several values of electron concentrations $n(n=5 / 4,3 / 2,7 / 4)$. To reveal the finite-size effects on the stability of ferromagnetic domains, the behavior of the critical interaction strength $U_{c}(q)$ has been calculated on several finite clusters at each electron filling. It is seen that finite-size effects on $U_{c}$ are small and thus these results can be satisfactorily extrapolated to the thermodynamic limit $L \rightarrow \infty$. Our results clearly demonstrate that the ferromagnetic state is strongly influenced by $q$ for electron concentrations above half-filling and generally it is stabilized with increasing $q$. The effect is especially strong for small values of $q$ where small changes of $q$ reduce dramatically the critical interaction strength $U_{c}$ and so the ferromagnetic state becomes stable for a wide range of model parameters. The results presented in figure $2 \mathrm{~d}$ show that only for $q=0$ (nearest-neighbor hopping) $U_{c}=\infty$, while for finite $q$ (that represents a much more realistic type of electron hopping), the critical interaction strength $U_{c}$ is finite. Thus, the absence of ferromagnetism in the ordinary Hubbard model with the nearest-neighbor hopping $(q=0)$ can be explained as a consequence of too simplified description of electron hopping on the lattice. For any $q>0$, ferromagnetism comes naturally from the Hubbard model with a generalized type of hopping for a wide range of model parameters without any other assumptions. This opens up a new route towards the understanding of ferromagnetism in the Hubbard model.
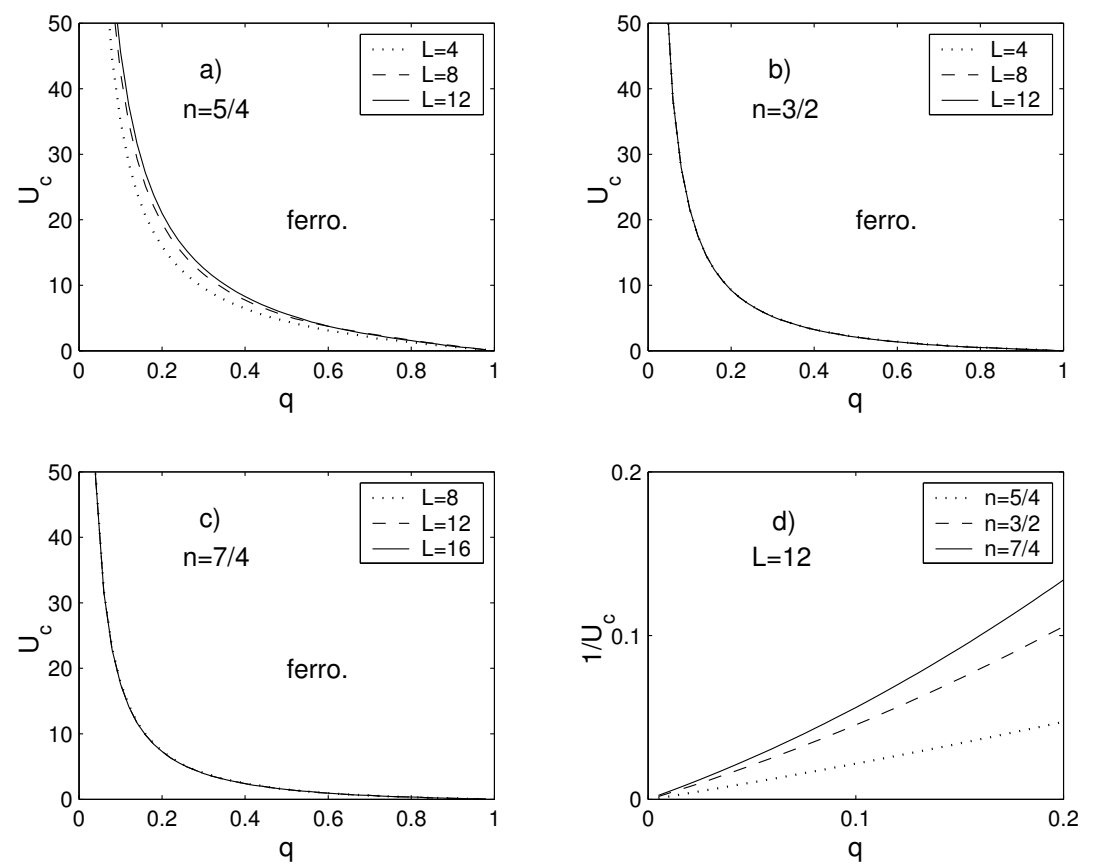

Figure 2. The critical interaction strength $U_{c}\left(1 / U_{c}\right)$ as a function of $q$ calculated for different $n$ and $L$ [31]. 

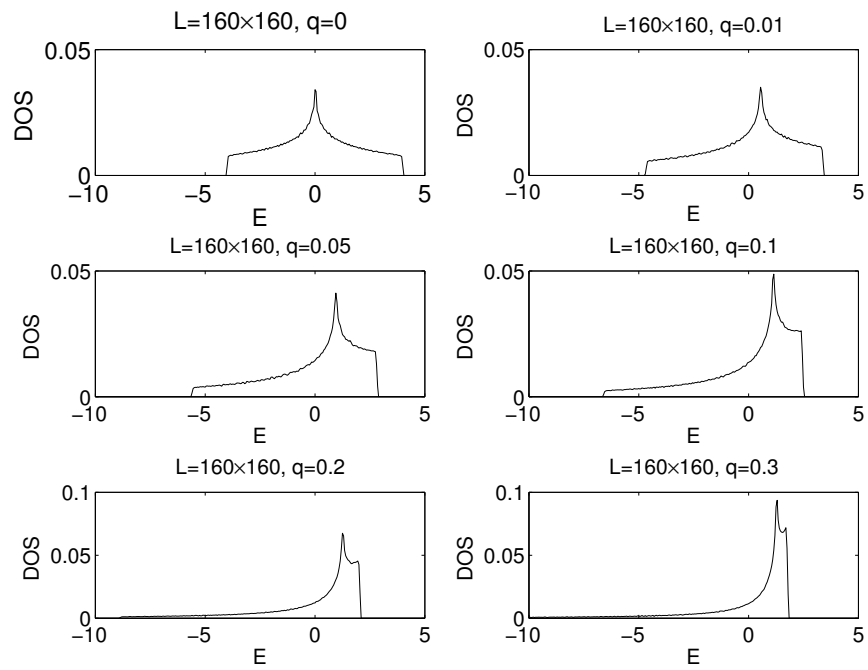

Figure 3. The non-interacting $(D=2)$ DOS corresponding to the long-range hopping for different $q$ and $L=160 \times 160$ [32].

\subsubsection{Two-dimensional case}

We have also performed the same calculations for the physically more interesting two-dimensional case [32]. Since in the two-dimensional case only very small clusters $(L \sim 20)$ are accessible by the exact diagonalization method, we support these calculations by the quantum Monte Carlo method that can treat several times larger clusters.

Similarly to the one-dimensional case, let us start with a discussion of long-range hopping effects on the noninteracting two-dimensional DOS (see figure 3). It is seen that with an increasing $q$, more weight shifts to the upper edge of the band and the DOS becomes strongly asymmetric, indicating possible ferromagnetic regions in the limit $n>1$ For this reason, we have focused our attention on the case of electron concentrations above half-filling $n>1$. First, we have examined the model Hamiltonian by the exact diagonalization method on the finite $4 \times 4$ cluster for several selected values of on-site Coulomb interaction $U(U=1,2,4,8)$ and electron concentrations $n>1(n=3 / 2$ and $n=7 / 4)$. The results of our numerical calculations are displayed in figure 4 There is plotted the difference $\Delta E=E_{f}-E_{g}$ between the exact ground state $E_{g}$ and the ferromagnetic state $E_{f}$ (which is exactly known) as a function of $1 / q$. The ground state is ferromagnetic in the regions where $\Delta E=0$. It can be seen that for higher electron concentrations $(n=7 / 4)$, the ground states of the two-dimensional Hubbard model with generalized hopping are non-ferromagnetic for all examined values of the on-site interaction $U$, which strongly contrasts with the one-dimensional case, where the ferromagnetic state has been stabilized for all electron concentrations above the half-filled band case $n>1$. However, for smaller values of electron concentrations $(n=3 / 2)$, the situation is fully different. In this case, the ferromagnetic state is the ground state of the model for all examined values of $U$ above some critical value of the long-range hopping parameter $q_{c}$. As shown in the inset in figure 4. $1 / q_{c}$ scales linearly with $U$, from which it can be directly determined that $1 / q_{c}=1.13+0.558 U$. Analysing these results one can see that already for relatively small values of the Coulomb interaction $U$, the critical values of the long-range hopping parameter $q_{c}$ are from the physically realistic regime (e.g., $q_{c} \cong 0.44$ for $U=2, q_{c} \cong 0.3$ for $U=4$ and $q_{c} \cong 0.18$ for $U=8$ ). This confirms the importance of the long-range electron hopping term for a correct description of ferromagnetism in real materials.

Since our numerical results revealed a strikingly different behaviour of the model in one and two dimensions for electron concentrations above half filling, and namely, the existence of the critical electron concentration $n_{c}$ below (above) which the ground state is ferromagnetic (non-ferromagnetic), we have 

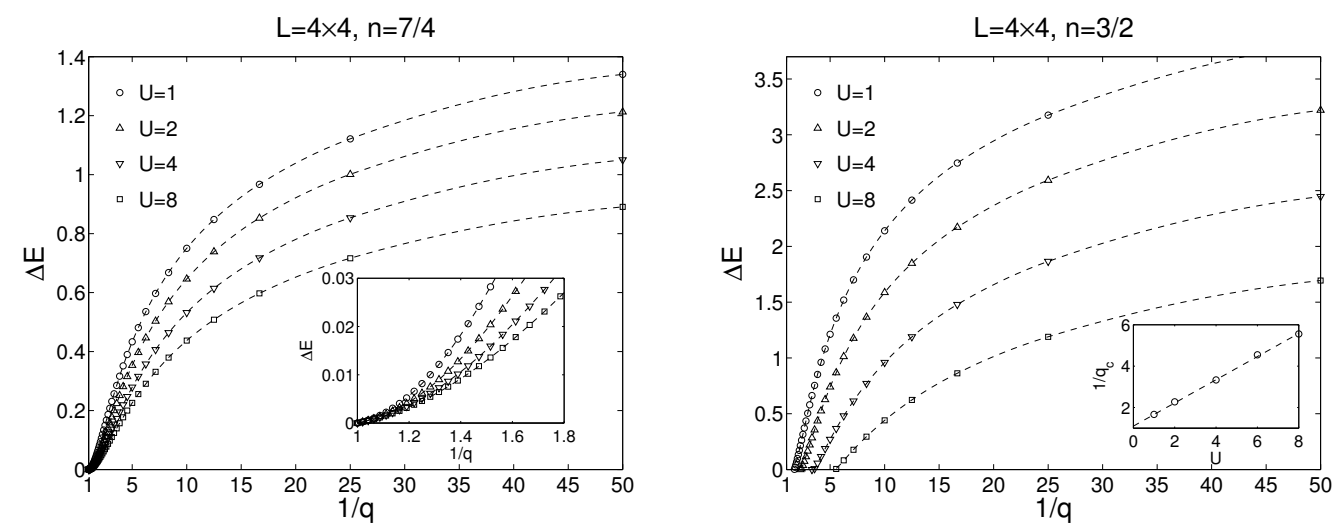

Figure 4. The left-hand panel: The difference $\Delta E=E_{f}-E_{g}$ between the exact ground state $E_{g}$ and the ferromagnetic state $E_{f}$ (which is exactly known since the state has no double hole occupancy) as a function of $1 / q$ calculated for $n=7 / 4$ and different $U$ on the $L=4 \times 4$ cluster. The inset shows the situation in the limit $1 / q \rightarrow 1$. The right-hand panel: $\Delta E=E_{f}-E_{g}$ as a function of $1 / q$ calculated for $n=3 / 2$ and different $U$ on the $L=4 \times 4$ cluster. The inset shows the $U$ dependence of $1 / q_{c}$ [32].

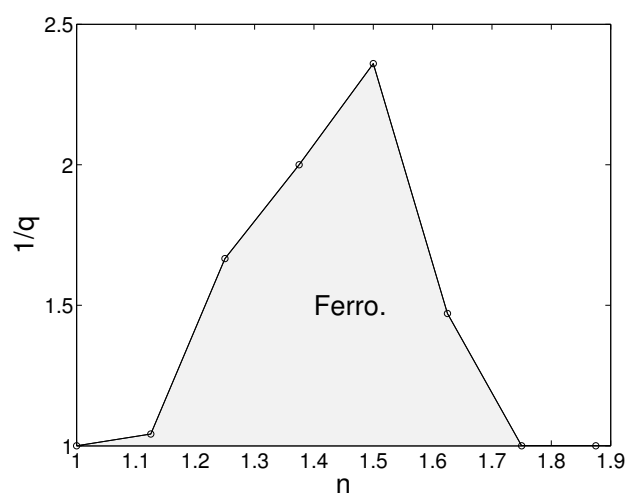

Figure 5. The phase diagram of the two-dimensional Hubbard model with long-range hopping in the $1 / q-n$ plane calculated for $U=2$ and $L=4 \times 4$ [32].

decided to study in detail the comprehensive phase diagram of the model in the $q-n$ plane. Again we have used the small cluster exact diagonalization technique and the cluster of $L=4 \times 4$ sites. The results of our numerical calculations obtained for the intermediate value of the Coulomb interaction $U=2$ are summarized in figure 5 They show that just $n=7 / 4$ is the critical electron concentration above which the ground state is non-ferromagnetic. Below this value, the ground state is ferromagnetic. The critical value of the inverse long-range hopping parameter $1 / q_{c}$ increases with an decreasing $n$ and reaches its maximum $\left(1 / q_{c} \sim 2.4\right)$ at $n=3 / 2$. A further decrease in $n$ gradually reduces the critical value of $1 / q_{c}$ to one. A similar behaviour of the model has been observed for both smaller $(U=1)$ as well as larger $(U=4)$ values of the Coulomb interaction. Thus, we can conclude that the ferromagnetic phase in the two dimensional Hubbard model, in spite of its partial reduction (in comparison to the one dimensional case), remains robust.

Unfortunately, these results cannot be considered as definite, since they were obtained on a very small cluster and, therefore, it is necessary to prove them independently by other methods. To fulfill this goal, we performed the same calculations for one representative value of $U(U=2)$ and for two representative values of $n(n=3 / 2,5 / 4)$ by the projector quantum Monte Carlo (QMC) method [28-30] that is capable of treating several times larger clusters with high accuracy. The QMC simulations were performed using a 

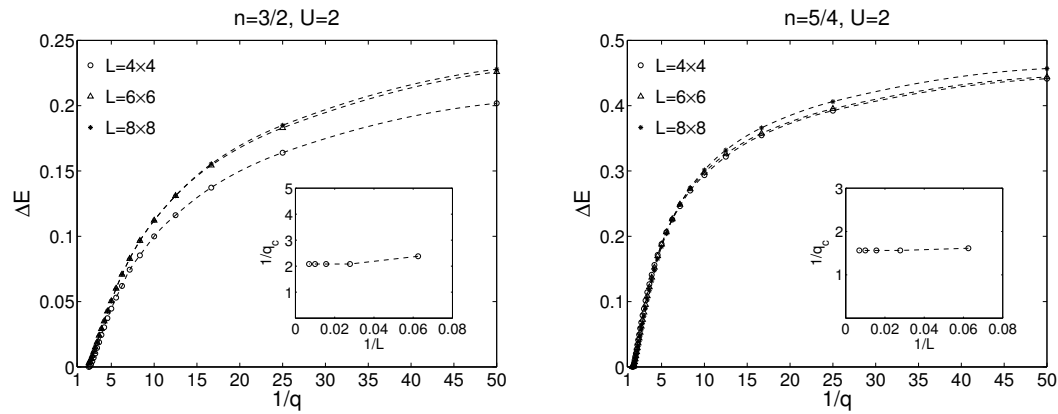

Figure 6. The difference $\Delta E=E_{f}-E_{g}$ between the ground state $E_{g}$ and the ferromagnetic state $E_{f}$ as a function of $1 / q$ calculated for $U=2$, two different values of $n(n=3 / 2$ and $n=5 / 4)$ and three different finite clusters of $L=4 \times 4, L=6 \times 6$ and $L=8 \times 8$. The QMC results [32].

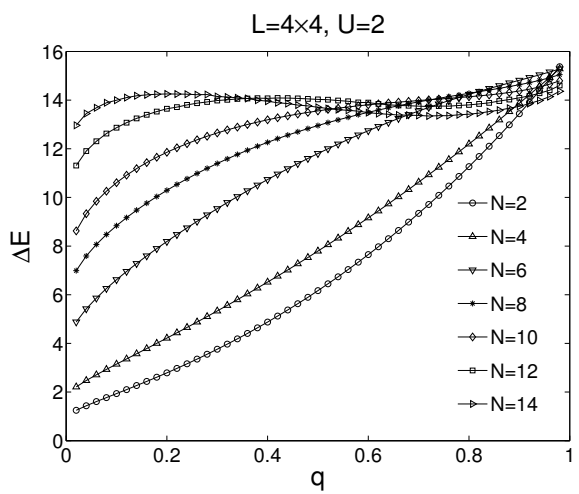

Figure 7. The difference $\Delta E=E_{f}-E_{g}$ between the exact ground state $E_{g}$ and the ferromagnetic state $E_{f}$ as a function of $q$ calculated for $U=2$ and different electron fillings $N$ on the $L=4 \times 4$ cluster [32].

projector algorithm which applies $\exp (-\theta H)$ to a trial wave-function (in our case, the solution for $U=0$ ). A projector parameter $\theta \sim 30$ and a time slice of $\Delta \theta=0.05$ suffice to reach well converged values of the observables discussed here.

The results of numerical calculations performed on larger clusters consisting of $L=6 \times 6$ and $L=8 \times 8$ sites (over the whole interval $[0,1]$ of $q$ values with the step $\Delta q=0.02$ ) and clusters of $L=10 \times 10$ and $L=12 \times 12$ sites (over a restricted set of $q$ values near $q_{c}$ ) are displayed in figure 6 . These results clearly show that the finite size effects on the critical values of the long-range hopping parameter are negligible (see insets in figure 6), and thus, the magnetic phase diagram found for the $4 \times 4$ cluster can be satisfactorily extrapolated on macroscopic systems.

Finally, let us briefly discuss the numerical results obtained for electron concentrations less than the half-filled band case $(n=1)$. They are displayed in figure 7 for $U=2$ and the complete set of even electron fillings with $N<L$ on the $4 \times 4$ cluster. These results clearly demonstrate the absence of the ferromagnetic ground state in the two-dimensional Hubbard model with exponentially decaying hopping amplitudes for all values the hopping parameter $q$ and electron concentrations $n<1$, showing a key role of the band filling $n$ in the mechanism of stabilization of the ferromagnetic state.

\subsection{The effect of correlated hopping}

Let us now further generalize the single-band Hubbard model with long-range hopping by introducing the correlated hopping term [40-43], in which the $\sigma$-electron hopping amplitudes between lattice sites $i$ 

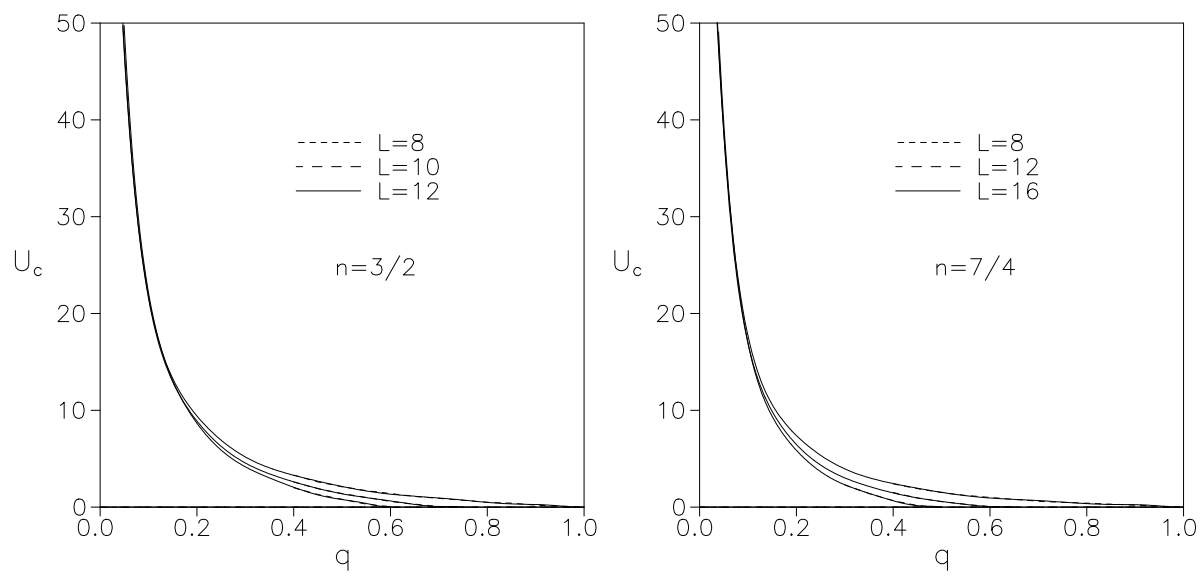

Figure 8. The critical interaction strength $U_{c}$ as a function of $q$ calculated for different $t^{\prime}$ and $L$ at $n=3 / 2$ (the left-hand panel) and at $n=7 / 4$ (the right-hand panel). Curves from up to down correspond to: $t^{\prime}=0,0.2$ and $0.4[34]$.

and $j$ depend explicitly on $n_{i-\sigma}$ and $n_{j-\sigma}$ occupancy, i.e.,

$$
t_{i j}^{\sigma}=t_{i j}\left[1+t^{\prime}\left(n_{i-\sigma}+n_{j-\sigma}\right)\right] .
$$

The importance of the action of the correlated hopping term on the ground-state properties of the Hubbard model has been already mentioned by Hubbard [1]. Later, Hirsch [44] pointed out that this term may be relevant in explaning the superconducting properties of strongly correlated electrons. Here, we discuss the effects of this term on the stability of the fully polarized ferromagnetic state. The same subject was studied by Amadon and Hirch [45], as well as by Kollar and Vollhardt [46], though they considered the hopping only between the nearest-neighbor sites.

The Hamiltonian of the single-band Hubbard model in which the effects of long-range and correlated hopping are incorporated is given by

$$
H=\sum_{i j \sigma} t_{i j}^{\sigma} c_{i \sigma}^{+} c_{j \sigma}+U \sum_{i} n_{i \uparrow} n_{i \downarrow}
$$

To examine the possibilities for the existence of ferromagnetism in this model, the ground states are determined by exact diagonalizations for a wide range of model parameters $\left(q, t^{\prime}, U, N=\sum_{\sigma} N_{\sigma}\right)$. Typical examples are then chosen from a large number of available results to represent the most interesting cases. The results of our small-cluster exact-diagonalization calculations obtained on finite clusters up to $L=16$ sites are summarized in figure 8 . There is shown the critical interaction strength $U_{c}$, above which the ground state is ferromagnetic, as a function of $q$ for the selected values of $n=N / L$ and $t^{\prime}$ $\left(n=3 / 2,7 / 4 ; t^{\prime}=0,0.2,0.4\right)$. To reveal the finite-size effects on the stability of ferromagnetic domains, the behavior of the critical interaction strength $U_{c}(q)$ was calculated on several finite clusters at each electron filling. It is seen that finite-size effects on $U_{c}$ are small and thus these results can be satisfactorily extrapolated to the thermodynamic limit $L \rightarrow \infty$. Our results clearly demonstrate that the ferromagnetic state is strongly influenced by correlated hopping $\left(t^{\prime}\right)$ and generally it is stabilized with increasing $t^{\prime}$. The effect is especially strong for intermediate and strong values of $q$. There even exists some critical value of $q$ above which the ground state is ferromagnetic for all nonzero $U$. With an increasing $t^{\prime}$, this critical value shifts to lower values of $q$ (that represent a much more realistic type of electron hopping) and the ferromagnetic domain correspondingly increases. Performing exhaustive numerical studies of the model for a wide range of electron concentrations (on different lattice clusters) we have found that the model exhibits the same behavior for all electron concentrations above half-filling] and that with an increasing

\footnotetext{
${ }^{1} n \leqslant 1$ and $t^{\prime}<0$ does not stabilize the ferromagnetic state.
} 
concentration this effect becomes more pronounced. These results clearly show that ferromagnetism comes naturally from the Hubbard model with long-range and correlated hopping for a wide range of model parameters without any other assumptions.

\subsection{The effect of long-range Coulomb interaction}

The microscopic model of electronic correlations in solids based on the picture of long-range electron hopping with exponentially decaying hopping amplitudes in combination with the standard on-site description of the Coulomb interaction between electrons on a lattice does not model very realistically the situation in real transition metal compounds. Indeed, it would be more correct to describe the Coulomb interaction between electrons by a similar formula that corresponds to the electron hopping [equation [2.1] ], i.e., by exponentially decaying interaction amplitudes $U_{i j}$,

$$
U_{i j}\left(q_{U}\right)= \begin{cases}U, & i=j, \\ \frac{U}{2} q_{U}^{|i-j|}, & i \neq j,\end{cases}
$$

where $U$ is the strength of the on-site Coulomb interaction and $q_{U}\left(0 \leqslant q_{U} \leqslant 1\right)$ is the parameter of the long-range Coulomb interaction. Such a selection is natural, since the overlap of the atomic wave functions decreases exponentially with a distance of two atoms on a lattice and thus the model based on such a supposition describes more realistically the situation in transition metal compounds.

Thus, our generalized Hamiltonian taking into account the effects of the long-range electron hopping as well as the effects of the long-range Coulomb interaction, both in the form of exponentially decaying amplitudes, can be written as follows:

$$
H=\sum_{i j \sigma} t_{i j}\left(q_{t}\right) c_{i \sigma}^{+} c_{j \sigma}+\sum_{i j \sigma \sigma^{\prime}} U_{i j}\left(q_{U}\right) n_{i \sigma} n_{j \sigma^{\prime}} .
$$

To reveal the behaviour of the model in different concentration limits, we selected three different values of the electron concentration, and namely, $n=1 / 2, n=1$ and $n=3 / 2$ that represent three physically most interesting limits of the model. Let us start the discussion of our results with the half-filled band case $n=1$. To see the pure effects of the long-range Coulomb interaction on the stability of the ferromagnetic state, we first examined the conventional limit of the electron hopping corresponding to the following selection of the hopping amplitudes $t_{i j}=-1$ if $i$ and $j$ are the nearest neighbour and $t_{i j}=0$ otherwise. For this case, we calculated the ground states of the model $E_{g}\left(N_{\uparrow}\right)$ in all different spin sectors $S_{z}=N_{\uparrow}-N_{\downarrow}$ as a function of $U$ and $q_{U}$ with steps $\Delta U=0.2$ and $\Delta q_{U}=0.02$. The resulting behaviours of $E_{g}\left(N_{\uparrow}\right)$ as functions of $q_{U}$ and $U$ are used directly to identify the stability regions of the fully polarized state, i.e., where the following inequality $E_{g}(0)<\min \left[E_{g}(1), E_{g}(2), \ldots, E_{g}(N / 2)\right]$ is satisfied for a given $U$ and $q_{U}$. The results of our numerical simulations obtained for $L=12, n=1$ and four different values of $U$ are shown in figure 9 (the left-hand panels). One can see that for each selected value of the Coulomb interaction $U$, there exists a critical value of the interaction parameter $q_{U}$ above which the ground state is the fully polarized state, i.e., $N_{\uparrow}=0, N_{\downarrow}=L$. Since the ground state of the ordinary (nearest-neighbour) half-filled Hubbard model in one dimension is antiferromagnetic for all Coulomb interactions, our results point to the crucial role of the long-range interaction on the stabilization of the ferromagnetic state. Moreover, we have found that the critical value of $q_{U}$ above which the ground state is ferromagnetic decreases rapidly with an increasing $U$ and thus already small, but physically the most realistic values of $q_{U}$, are capable of generating the ferromagnetic state for intermediate and strong Coulomb interactions. The same conclusions are also valid for electron concentrations smaller than the half-filled band case $(n<1)$, as illustrates figure 9 (the right-hand panels) for the quarter-band filling $n=1 / 2$, while in the opposite limit $n>1$ no effects of long-range interaction on the stabilization of the band ferromagnetism are observed. Comparing these results with the ones discussed above for $q_{U}=0$ and $q_{t}>0$, one can find a fundamentally different response of the electron system to the long-range electron hopping and the long-range Coulomb interaction. Indeed, while the long-range electron hopping itself, i.e., without the long-range Coulomb interaction, stabilizes the ferromagnetic state for the electron concentrations above 

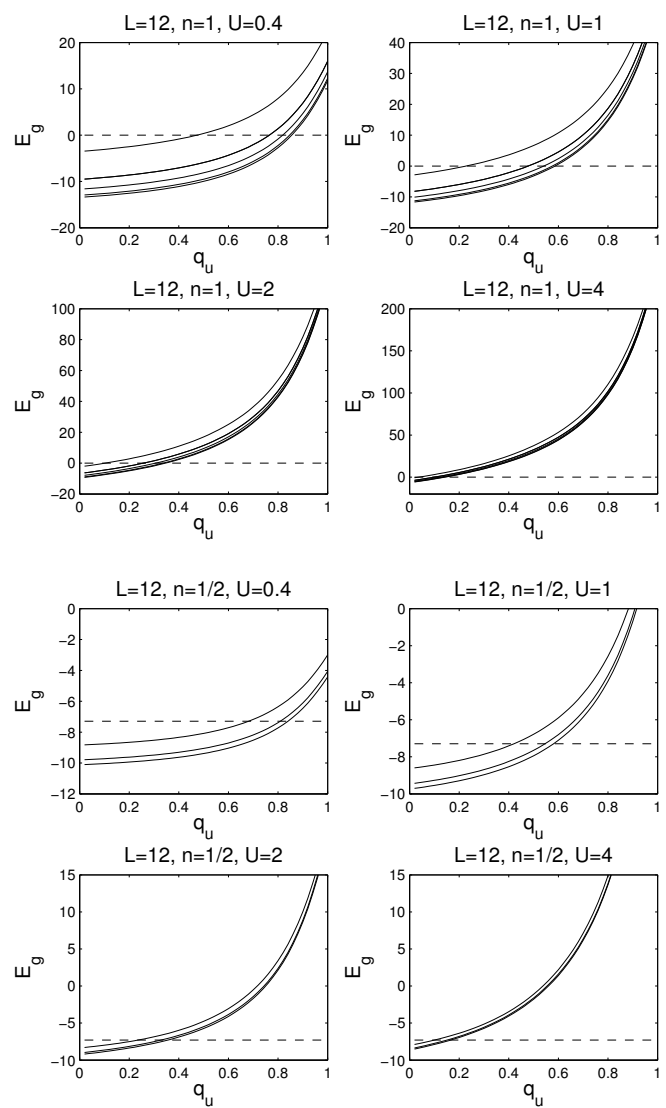

Figure 9. The left-hand panel: The ground-state energy $E_{g}$ as a function of $q_{U}$ calculated for $q_{t}=0, L=$ $12, n=1$ and different values of $U$ and $N_{\uparrow}$. The dashed line corresponds to $N_{\uparrow}=0$ (the fully polarized state) and the full lines (from up to down) correspond to $N_{\uparrow}=1,2,3,4,5$ (the partially polarized states) and $N_{\uparrow}=6$ (the non-polarized state). The right-hand panel: The ground-state energy $E_{g}$ as a function of $q_{U}$ calculated for $q_{t}=0, L=12, n=1 / 2$ and different values of $U$ and $N_{\uparrow}$. The dashed line corresponds to $N_{\uparrow}=0$ (the fully polarized state) and the full lines (from up to down) correspond to $N_{\uparrow}=1,2$ (the partially polarized states) and $N_{\uparrow}=3$ (the non-polarized state) [33].

the half-filled band case $(n>1)$, the long-range Coulomb interaction itself stabilizes the ferromagnetic state in the opposite limit, i.e., for $n \leqslant 1$. Thus, one can intuitively expect that the combined effects of the long-range electron hopping and the long-range Coulomb interaction could lead to the stabilization of the ferromagnetic state for the electron concentrations smaller as well as larger than the half-filled band case.

To verify this conclusion, we performed exhaustive numerical studies of the model for a wide range of the model parameters. In particular, we selected five different values of the long-range hopping parameter $q_{t}>0\left(q_{t}=0.1,0.2,0.3,0.4,0.5\right)$ and for each of them we calculated the ground-state energy $E_{g}\left(N_{\uparrow}\right)$ in all different spin sectors, for the following sequences of $q_{U}$ and $U$ values: $q_{U}=0,0.02, \ldots, 1$ and $U=0,0.2, \ldots, 30$. The results obtained are summarized in the form of the $U-q_{U}$ magnetic phase diagram, where the critical interaction strength $U_{c}\left(q_{U}\right)$ represents the phase boundary above which the ground state is a fully polarized state. The situation for the quarter-band filling is shown in figure 10 (the left-hand panel). Our results clearly demonstrate that the ferromagnetic state is strongly influenced by $q_{U}$ for electron concentrations bellow half-filling and generally it is stabilized with an increasing $q_{U}$. The effect is especially strong for small values of $q_{U}$ where small changes of $q_{U}$ reduce dramatically the critical interaction strength $U_{c}$ and so the ferromagnetic state becomes stable for a wide range of Coulomb interaction $U$. The second general trend that can be deduced from our numerical calculations is 


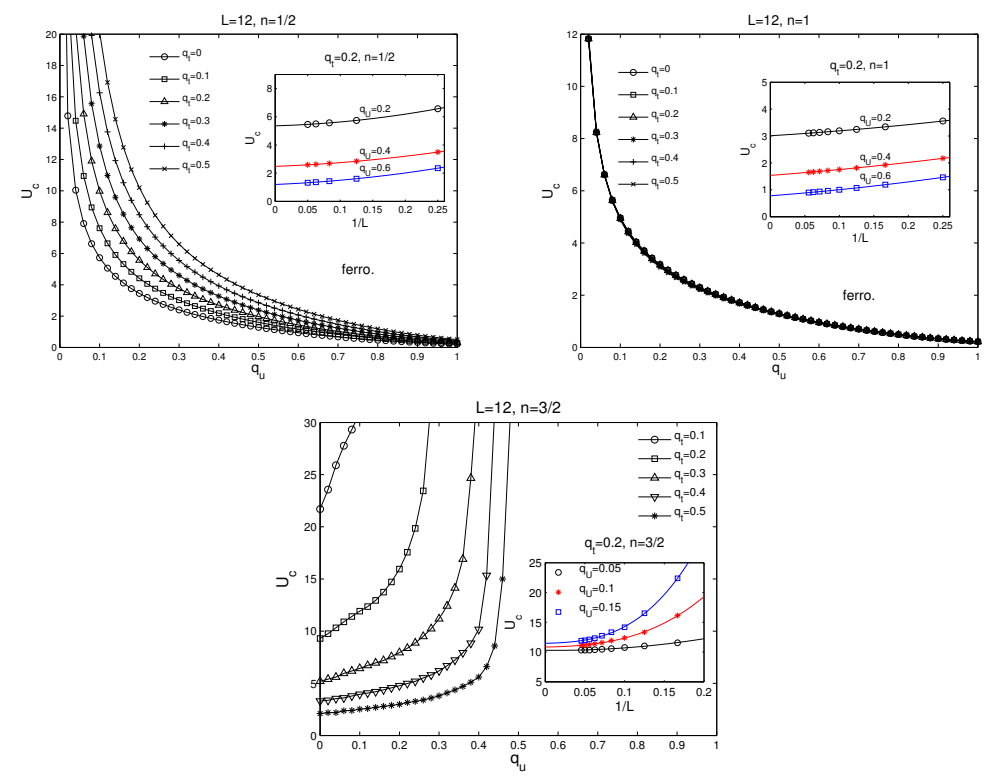

Figure 10. (Colour online) The left-hand panel: The critical interaction strength $U_{c}$ as a function of $q_{U}$ calculated for $L=12, n=1 / 2$ and different values of $q_{t}$. The inset shows the scaling of $U_{c}$ calculated for three different values of $q_{U}$. The scaling function is the second order polynomial expression in 1/L. The middle panel: $U_{c}$ as a function of $q_{U}$ calculated for $L=12, n=1$ and different values of $q_{t}$. The inset shows scaling $U_{c}$ calculated for three different values of $q_{U}$. The scaling function is the second order polynomial expression in $1 / L$. The right-hand panel: $U_{c}$ as a function of $q_{U}$ calculated for $L=12, n=3 / 2$ and different values of $q_{t}$. The inset shows the scaling of $U_{c}$ calculated for three different values of $q_{U}$. The scaling function is the third order polynomial expression in $1 / L[33]$.

depicted by the ferromagnetic domain being reduced with an increasing $q_{t}$, though it remains robust for a wide range of $q_{U}$ and $U$ values. For $n<1$, this result is intuitively expected since the long-range hopping itself stabilizes the ferromagnetic state only for $n>1$. To exclude the finite-size effects on the stability of ferromagnetic domains we performed an exhaustive numerical study of the $L$-dependence of the critical interaction strength $U_{c}$ for selected values of $q_{t}$ and $q_{U}$ on finite clusters from $L=4$ to $L=20$. The inset in figure 10 (the left-hand panel) shows that finite-size effects on $U_{c}$ are small for all examined values of $q_{U}$ and thus these results can be satisfactorily extrapolated to the thermodynamic limit $L \rightarrow \infty$.

We also obtained similar results for the half-filled band case $n=1$ (see figure 10 , the middle panel). However, in this case the critical interaction strength $U_{c}\left(q_{U}\right)$ exhibits a universal behaviour, i.e., it does not depend on the value of the long-range hopping parameter $q_{t}$. Since in the half-filled band case and $q_{U}=0$, the ground states of the ordinary $\left(q_{t}=0\right)$ as well as the generalized $\left(q_{t}>0\right)$ Hubbard model are non-ferromagnetic [31], the existence of the ferromagnetic state at this filling is purely a consequence of long-range interactions. Moreover, analysing the behaviour of $U_{c}\left(q_{U}\right)$ for $q_{U} \rightarrow 0$, we found that only for $q_{U}=0$ (the Hubbard model with one-site interaction) $U_{c}=\infty$, while for finite $q_{U}$ (that represents a much more realistic type of interactions between electrons on a lattice), the critical interaction strength $U_{c}$ is finite. Thus, the absence of ferromagnetism in an ordinary model as well as in a generalized Hubbard model with long-range hopping at $n \leqslant 1$ could be explained as a consequence of a too simplified description of electron-electron interactions on the lattice. For any $q_{U}>0$, ferromagnetism comes naturally from the Hubbard model with long-range interactions for a wide range of model parameters without any other assumptions. As it is shown in the inset in figure 10 (the middle panel), our numerical results obtained for $n=1$ depend only very weakly on the size of the lattice and thus they can be satisfactorily extrapolated to macroscopic systems.

Above the half-filled band case $n>1$, we expect a strong interplay between the effects of long-range hopping and long-range interaction, since the long-range hopping itself stabilizes the ferromagnetic state 
for $n>1$ [31], while the long-range interaction itself produces a fully opposite effect. The results of our numerical simulations obtained for $n=3 / 2$ are presented in figure 10 (the right-hand panel) and they fully confirm this conjecture. Due to the combined effects of the long-range hopping and the long-range Coulomb interaction, the stability region of the ferromagnetic phase is reduced in comparison to the onsite case $\left(q_{U}=0\right)$, though it remains finite for all the examined values of $q_{t}$. Similarly to the preceding cases, the results can be satisfactorily extrapolated to the thermodynamic limit $L \rightarrow \infty$ and can be used for a description of macroscopic systems.

\subsection{The effect of flat bands}

\subsubsection{One-dimensional case}

In our previous paper we showed [47] that the ferromagnetic state can be also found in the so-called static limit of the Hubbard model, where only one kind of electrons, say with spins up, can move on the lattice, while electrons of the opposite spins are immobile. In this limit, the ferromagnetic state is stabilized for a wide range of the on-site Coulomb interaction $U$ between the up and down spin electrons for both the hole doped case (the total concentration of electrons $n<1)$ and for the electron doped case $(n>1)$. From this point of view, it is interesting to ask whether the ferromagnetic state found for the zero value of the down-spin electron hopping integral $t_{\downarrow}$ persists also at finite $t_{\downarrow}$, or vanishes discontinuously as soon as $t_{\downarrow}>0$. To answer this question, we numerically examined using the density matrix renormalization group (DMRG) method [25-27], the asymmetric Hubbard model [48-50] $\left(0 \leqslant t_{\downarrow} \leqslant 1\right)$ that incorporates both the full Hubbard model $t_{\uparrow}=t_{\downarrow}$ and its static $\left(t_{\downarrow}=0\right)$ limit (in the rare-earth community also known as the Falicov-Kimball model [51]).

The Hamiltonian of the asymmetric Hubbard model is

$$
H=-t_{\uparrow} \sum_{<i j>} c_{i \uparrow}^{+} c_{j \uparrow}-t_{\downarrow} \sum_{<i j>} c_{i \downarrow}^{+} c_{j \downarrow}+U \sum_{i} c_{i \uparrow}^{+} c_{i \uparrow} c_{i \downarrow}^{+} c_{i \downarrow},
$$

where $c_{i \uparrow}^{+}\left(c_{i \uparrow}\right)$ and $c_{i \downarrow}^{+}\left(c_{i \downarrow}\right)$ is the creation (annihilation) operator of light ( $\uparrow$ ) and heavy $(\downarrow)$ electron at the lattice site $i$.

The first two terms of (2.7) are the kinetic energies corresponding to quantum-mechanical hopping of up-spin and down-spin electrons between the nearest neighbor sites $i$ and $j$ with hopping probabilities $t_{\uparrow}$ and $t_{\downarrow}$, respectively. The third term represents the on-site Coulomb interaction between the up-spin electrons with density $n_{\uparrow}=\frac{1}{L} \sum_{i} d_{i \uparrow}^{+} d_{i \uparrow}$ and the down-spin electrons with density $n_{\downarrow}=\frac{1}{L} \sum_{i} d_{i \downarrow}^{+} d_{i \downarrow}$. The model is called "asymmetric" because the hopping integrals for up-spin and down-spin electrons may be different. Usually, the hopping integral of the up-spin electrons is taken to be the unit of energy $\left(t_{\uparrow}=1\right)$ and the down-spin-electron hopping integral is considered in the limit $t_{\downarrow} \leqslant 1$. This is the reason why the up-spin electrons are called light ones and the down-spin electrons are called heavy.

Based on the results obtained in the static limit of the model [47], we chose three different concentration limits, and namely, $n=1 / 4,1 / 2$ and $3 / 4$, at which we performed exhaustive numerical studies of the asymmetric Hubbard model, with a goal to reveal the effects of the down-spin electron hopping on the stability of the ferromagnetic state found at $t_{\downarrow}=0$. In all three limits, the comprehensive magnetic phase diagrams of the model in the $t_{\downarrow}-U$ plane are constructed using the DMRG method, that allows us to treat relatively large clusters with high accuracy. To identify different magnetic phases that enter the ground-state phase diagrams, we calculated the DMRG ground-state energy $E_{\mathrm{DMRG}}$, the total spin $S$ in the DMRG ground state by evaluating

$$
\left\langle\mathbf{S}^{\mathbf{2}}\right\rangle=\sum_{\mathbf{i}, \mathbf{j}}\left\langle\mathbf{S}_{\mathbf{i}} \mathbf{S}_{\mathbf{j}}\right\rangle
$$

and the energy of the fully polarized state $E_{f}$, which is exactly known since the state has no double occupancy. Different phases are then classified as follows: (i) the fully polarized state, $E_{\mathrm{DMRG}}>E_{f}$ and $S=S_{\max }$, (ii) the partially polarized state, $E_{\mathrm{DMRG}}<E_{f}$ and $S \neq S_{\max }$ and (iii) the non-polarized state, $E_{\mathrm{DMRG}}<E_{f}$ and $S=0$. 
The resultant magnetic phase diagrams are presented in figure 11 . For small electron concentrations $(n=1 / 4)$, the ferromagnetic domain takes the largest part of the phase diagram. The ferromagnetic state is stable for all values of the down-spin electron hopping integrals $t_{\downarrow}>0.75$, and its stability region is further stabilized with an increasing Coulomb interaction $U$. The non-polarized state is stable only in a very narrow region near the full Hubbard limit $\left(t_{\uparrow}=t_{\downarrow}=1\right)$ and thereby it becomes extremely sensitive to small changes in $t_{\uparrow}$ and $t_{\downarrow}$. This is probably also the reason why the ferromagnetic state can be stabilized by the correlated hopping [45], when the original electron hopping amplitudes are reduced according to whether the sites $i$ and $j$ are occupied or are not occupied by the electron of the opposite spin. The qualitatively same picture is also observed for intermediate electron concentrations $(n=1 / 2)$, with only one exception, and namely, that the ferromagnetic phase is now reduced against the non-polarized and partially polarized phases. This trend also holds for higher electron concentrations $(n=3 / 4)$, when the ferromagnetic phase persists only in the strong coupling limit, exactly in accordance with conclusions obtained for the static limit $t_{\downarrow}=0$. In addition, using two complementary methods based on the calculations of the structure factors [52] and the most probable distribution of heavy particles [53] we found that with an exception of the partially polarized phase (at $n=3 / 4$ ), all remaining phases are homogeneous. The partially polarized phase found at $n=3 / 4$ is homogeneous only for sufficiently large $t_{\downarrow}$ while in the opposite limit, the phase separation takes place. This result accords with the ones obtained for the case of the asymmetric Hubbard model in which both $n_{\uparrow}$ and $n_{\downarrow}$ are fixed [52-54].
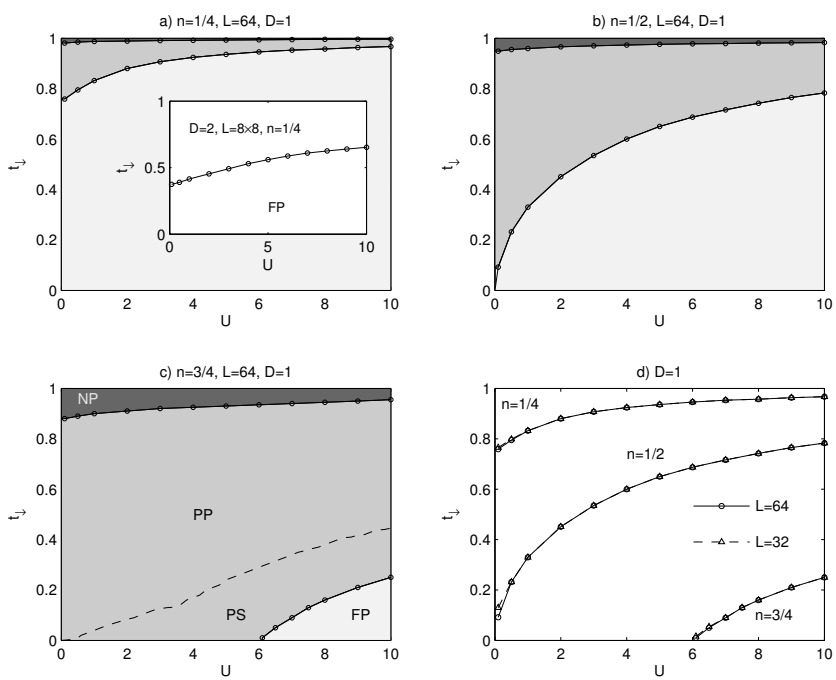

Figure 11. Phase diagrams of the asymmetric Hubbard model in $D=1$ calculated for three electron concentrations $(n=1 / 4, n=1 / 2$ and $n=3 / 4)$. Different phases correspond to the non-polarized NP phase (the black areas), the partially polarized PP phase (the gray areas) and the fully polarized FP phase (the light gray areas). In the PS region $(n=3 / 4)$, the ground states are phase separated. The inset in figure 11 a shows the results for $D=2$ obtained on $L=8 \times 8$ cluster. Figure $1 \mathrm{~d}$ presents finite-size effects on the phase boundary between the FP and PP phase in $D=1$ [35].

To exclude the influence of finite-size effects on the magnetic-phase diagrams of the asymmetric Hubbard model, we also calculated the phase boundaries for different finite clusters of $L=32$ and $L=64$ sites. The results of numerical calculations obtained for $n=1 / 4,1 / 2$ and $3 / 4$ are shown in figure $11 \mathrm{~d}$ and they clearly show that finite size effects are negligible and thus the results obtained can be satisfactorily extrapolated to the macroscopic systems. 

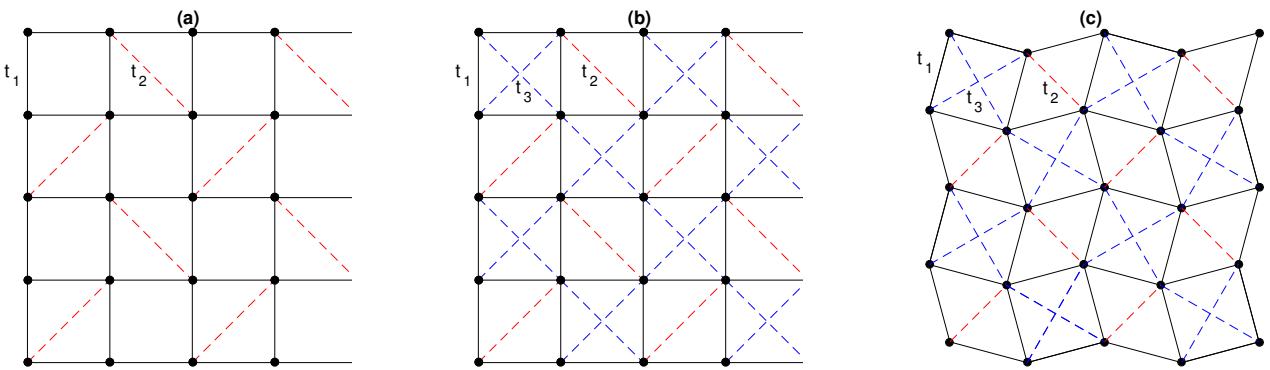

Figure 12. (Colour online) (a) The original SSL with the first $\left(t_{1}\right)$ and second $\left(t_{2}\right)$ nearest-neighbor couplings. (b) The generalized SSL with the first $\left(t_{1}\right)$, second $\left(t_{2}\right)$ and third $\left(t_{3}\right)$ nearest-neighbor couplings, and (c) the topologically identical structure realized in the (001) plane of rare-earth tetraborides [36].

\subsubsection{Two-dimensional case}

From the point of view of real materials, the question of fundamental importance is whether these results also persist in higher dimensions. For this reason, we also performed similar calculations in two dimensions. Since the DMRG method does not work very well in $D>1$, especially for the calculation of long-range correlation functions like $\left\langle\mathbf{S}_{\mathbf{i}} \mathbf{S}_{\mathbf{j}}\right\rangle$, instead of the DMRG method we used the projector quantum Monte Carlo method [28,-30]. The quantum Monte Carlo simulations were performed using a projector algorithm which applies $\exp (-\theta H)$ to a trial wave-function (in our case, the solution for $U=0$ ). A projector parameter $\theta \sim 30$ suffices to reach well converged values of the observables discussed here. A time slice of $\Delta \theta=0.05$ was used in general. The resultant numerical solutions for the critical values of the down-spin electron hopping integrals $t_{\downarrow}$ below which the ground state is ferromagnetic are shown in the inset in figure $11 \mathrm{~A}$. One can see that the ferromagnetic state is also robust in two dimensions although the corresponding values of $t_{\downarrow}$ in two dimensions are slightly smaller than the ones in $D=1$.

\subsection{Influence of lattice structure}

\subsubsection{Short-range hopping}

The results presented in the previous sections indicate that the lattice structure, which dictates the shape of the DOS, plays an important role in stabilizing the ferromagnetic state. Motivated by these results, we performed the same calculations on the special type of lattice, the so-called Shastry-Sutherland lattice (SSL). The SSL represents one of the simplest systems with geometrical frustration, so that putting the electrons on this lattice, one can simultaneously examine both the effect of interaction and the effect of geometrical frustration on the ground-state properties of the Hubbard model. This lattice was first introduced by Shastry and Sutherland [55] as an interesting example of a frustrated quantum spin system with an exact ground state. It can be described as a square lattice with the nearest-neighbor links $t_{1}$ and the next-nearest neighbors links $t_{2}$ in every second square (see figure $12 \mathrm{a}$ ). The SSL attracted much attention after its experimental realization in the $\mathrm{SrCu}_{2}\left(\mathrm{BO}_{3}\right)_{2}$ compound [56]. The observation of a fascinating sequence of magnetization plateaus (at $m / m_{s}=1 / 2,1 / 3,1 / 4$ and $1 / 8$ of the saturated magnetization $m_{s}$ ) in this material [57] stimulated further theoretical and experimental studies of the SSL. The SSL with the first, second and third nearest-neighbor links is shown in figure $12 \mathrm{p}$ and this is just the lattice that will be used in our next numerical calculations.

Thus, our starting Hamiltonian, corresponding to the one band Hubbard model on the SSL, can be written as follows:

$$
H=-t_{1} \sum_{\langle i j\rangle_{1}, \sigma} c_{i \sigma}^{+} c_{j \sigma}-t_{2} \sum_{\langle i j\rangle_{2}, \sigma} c_{i \sigma}^{+} c_{j \sigma}-t_{3} \sum_{\langle i j\rangle_{3}, \sigma} c_{i \sigma}^{+} c_{j \sigma}+U \sum_{i} n_{i \uparrow} n_{i \downarrow} .
$$

The first three terms of (1) are the kinetic energies corresponding to the quantum-mechanical hopping of electrons between the first, second and third nearest neighbors and the last term is the Hubbard on-site 

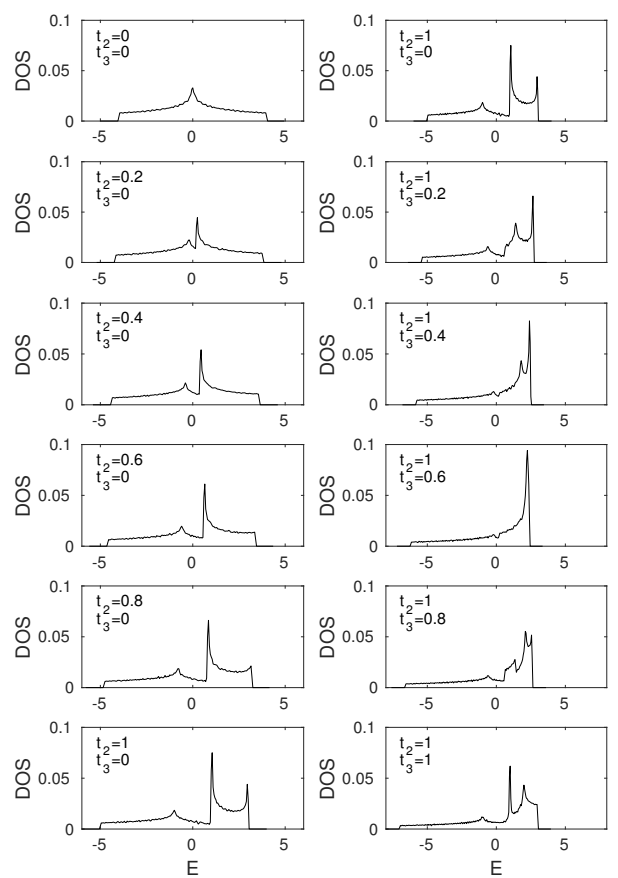

Figure 13. Non-interacting DOS calculated numerically for different values of $t_{2}$ and $t_{3}$ on the finite cluster of $L=200 \times 200$ sites [36].

repulsion between two electrons with opposite spins. We set $t_{1}=1$ as the energy unit and thus $t_{2}\left(t_{3}\right)$ can be seen as a measure of the frustration strength. It should be noted that most of the papers on the SSL concern various spin models, while there are only a few papers concerning the interacting electrons (the Hubbard model) on the SSL [58].

To identify the nature of the ground state of the Hubbard model on the SSL we have used the smallcluster-exact-diagonalization (Lanczos) method [24] and the projector quantum Monte Carlo method [30]. In both cases, the numerical calculations proceed in the following steps. Firstly, the ground-state energy of the model $E_{g}\left(S_{z}\right)$ is calculated in all different spin sectors $S_{z}=N_{\uparrow}-N_{\downarrow}$ as a function of the model parameters $t_{2}, t_{3}$ and $U$. Then, the resulting behaviors of $E_{g}\left(S_{z}\right)$ are used directly to identify the regions in the parametric space of the model, where the fully polarized state has the lowest energy. To reveal the possible stability regions of the ferromagnetic state in the Hubbard model on the SSL, let us first examine the effects of the geometrical frustration, represented by nonzero values of $t_{2}$ and $t_{3}$, on the behavior of the non-interacting DOS. The previous numerical studies of the standard one-dimentional and twodimensional Hubbard model with next-nearest [3] as well as long-range [31, 32] hopping showed that just this quantity could be used as a good indicator for the emergence of ferromagnetism in the interacting systems. The noninteracting DOS of the $U=0$ Hubbard model on the SSL of size $L=200 \times 200$, obtained by exact diagonalization of $H$ (for $U=0$ ) is shown in figure 13 . The left-hand panels correspond to the situation when $t_{2}>0$ and $t_{3}=0$, while the right-hand panels correspond to the situation when both $t_{2}$ and $t_{3}$ are finite. Numerical calculations were performed for different values of the delta function broadening $\epsilon$ and it was found that the value of $\epsilon=0.02$ (the case presented in this section) is sufficient to satisfactorily reproduce all significant features of DOS. One can see that once the frustration parameter $t_{2}$ is nonzero, the spectral weight starts to shift to the upper band edge and the noninteracting DOS becomes strongly asymmetric. Thus, taking into account the above mentioned scenario, there is a real chance that the interacting system could be ferromagnetic in the limit of high electron concentrations. To verify this conjecture, we performed exhaustive numerical studies of the model Hamiltonian (2.9) for a wide range 

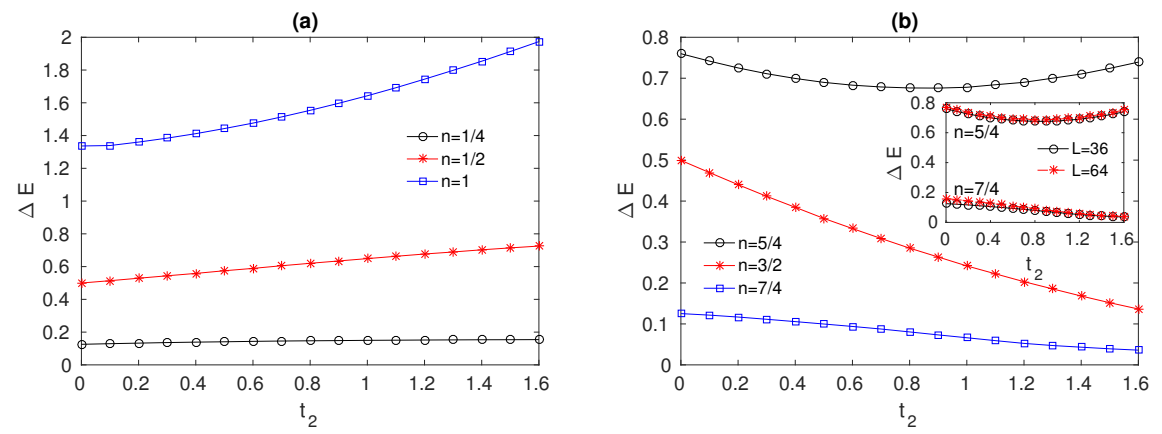

Figure 14. (Colour online) The difference $\Delta E=E_{f}-E_{\min }$ between the ferromagnetic state $E_{f}$ and the lowest ground-state energy from $E_{g}\left(S_{z}\right)$ as a function of the frustration parameter $t_{2}$ calculated for $n \leqslant 1$ (a) and $n>1$ (b) on the finite cluster of $L=6 \times 6$ sites $\left(U=1, t_{3}=0\right)$. The inset shows $\Delta E$, calculated for two different electron densities on clusters of $L=6 \times 6$ and $L=8 \times 8$ sites [36].

of the model parameters $U, t_{2}$ and $n$ at $t_{3}=0$. Typical results of our PQMC calculations obtained on finite cluster of $L=6 \times 6$ sites, in two different concentration limits $(n \leqslant 1$ and $n>1)$ are shown in figure 14. There is plotted the difference $\Delta E=E_{f}-E_{\min }$ between the fully saturated ferromagnetic state $E_{f}$, which can be calculated exactly and the lowest ground-state energy from $E_{g}\left(S_{z}\right)$ as a function of the frustration parameter $t_{2}$. According to this definition, the ferromagnetic state, strictly referred to as the fully saturated ferromagnetic state, corresponds to $\Delta E=0$ (everywhere in the paper, the notation ferromagnetism (ferromagnetic state) concerns the fully saturated ferromagnetism (the fully saturated ferromagnetic state)). It is seen that for electron concentrations below the half filled band case $n=1, \Delta E$ is the increasing function of $t_{2}$, and thus there is no sign of stabilization of the ferromagnetic state for $n \leqslant 1$, in accordance with the above mentioned scenario.

The situation looks more promising in the opposite limit $n>1$. In this case, $\Delta E$ is considerably reduced with an increasing $t_{2}$, though this reduction is still insufficient to reach the ferromagnetic state $\Delta E=0$ for physically reasonable values of $t_{2}\left(t_{2}<1.6\right)$ that correspond to the situation in the real materials. To exclude the finite-size effect, we also performed the same calculations on the larger cluster of $L=8 \times 8$ sites, but again no signs of stabilization of the ferromagnetic state were observed (see the inset to figure $14 \mathrm{~b}$ ).

For this reason, we turned our attention to the case $t_{2}>0$ and $t_{3}>0$. The noninteracting DOS corresponding to this case is displayed in figure 13 (the right-hand panels). These panels clearly demonstrate that with the increasing value of the frustration parameter $t_{3}$, a more spectral weight is still shifted to the upper band edge. A special situation arises at $t_{3}=0.6$, when the spectral weight is strongly peaked at the upper band edge. In this case, the nonintercting DOS is practically identical to the one corresponding to noninteracting electrons with long-range hopping [31, 32]. Since the long-range hopping supports ferromagnetism in the standard Hubbard model for electron concentrations above the half-filled band case [31, 32], we expect that this could be also true for the Hubbard model on the SSL, at least for some values of frustration parameters $t_{2}$ and $t_{3}$. Therefore, we decided to perform numerical studies of the model for a wide range of $t_{3}$ values at fixed $t_{2}, U$ and $n\left(t_{2}=1, U=1, n=7 / 4\right)$. To minimize the finite-size effects, the numerical calculations were done on two different finite clusters of $L=6 \times 6$ and $L=8 \times 8$ sites. The results of our calculations for $\Delta E$ as a function of $t_{3}$ are displayed in figure 15 a. In accordance with the above mentioned assumptions, we find a relatively wide region of $t_{3}$ values around $t_{3}=0.6$, where the ferromagnetic state is stable. It is seen that the finite-size effects on the stability region of the ferromagnetic phase are negligible and thus these results can be satisfactorily extrapolated to the thermodynamic limit $L=\rightarrow \infty$. Moreover, the same calculations performed for different values of the Hubbard interaction $U$ showed that the correlation effects (nonzero $U$ ) further stabilize the ferromagnetic state and lead to the emergence of macroscopic ferromagnetic domain in the $t_{3}-U$ phase diagram (see inset to figure $15 \mathrm{p}$ ). This confirms the crucial role of the Hubbard interaction $U$ in the mechanism of stabilization of ferromagnetism on the geometrically frustrated lattice. In figure $15 \mathrm{~b}$, we plotted the com- 

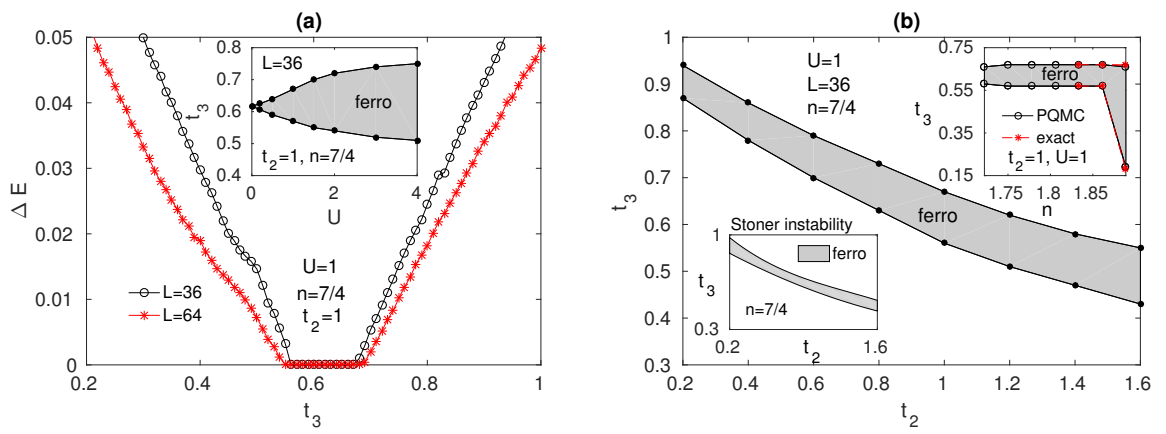

Figure 15. (Colour online) (a) The difference $\Delta E=E_{f}-E_{\min }$ as a function of the frustration parameter $t_{3}$ calculated for $U=1, t_{2}=1, n=7 / 4$ and two different finite clusters of $L=6 \times 6$ and $L=8 \times 8$ sites. The inset shows the ground-state diagram of the model in the $t_{3}-U$ plane. (b) The comprehensive phase diagrams of the model in the $t_{3}-t_{2}$ and $t_{3}-n$ plane [36].

prehensive phase diagrams of the model in the $t_{3}-n$ as well as $t_{3}-t_{2}$ plane, which clearly demonstrate that the ferromagnetic state is robust with respect to doping and frustration. In addition, we also calculated the stability region of the ferromagnetic state using the well-known Stoner criterion, and surprisingly, we found a nice correspondence of the results over the whole region of $t_{2}$ and $t_{3}$ values (see the lower inset to figure $15 \mathrm{p}$ ), despite the fact that the Stoner criterion is in general a crude approximation.

To check the convergence of PQMC results, we performed the same calculations by the Lanczos exact diagonalization method. Of course, on such a large cluster, consisting of $L=6 \times 6$ sites, we were able to examine (due to high memory requirements) only several electron fillings near the fully occupied band $(N=2 L)$. The exact diagonalization and PQMC results for the width of the ferromagnetic phase obtained on finite cluster of $L=6 \times 6$ sites, for three different electron fillings from the high concentration limit $(N=66,67,68)$, are displayed in the inset to figure $15 \mathrm{p}$ and they show a nice convergence of PQMC results.

Let us finally turn our attention to the question of possible connection between ferromagnetism and the noninteracting DOS that is discussed at the beginning of the paper. Figure $15 \mathrm{a}$ and figure $15 \mathrm{~b}$ show that for each finite $U$ and $n$ sufficiently large $(n \sim 7 / 4)$, there exists a finite interval of $t_{3}$ values, around $t_{3} \sim 0.6$, where the ferromagnetic state is the ground state of the model. To examine a possible connection between ferromagnetism and the noninteracting DOS, we numerically calculated the noniteracting DOS for several different values of $t_{3}$ from this interval and its vicinity. The results obtained for $U=1, n=7 / 4$ and $t_{2}=1$ are displayed in figure 16 Comparing these results with the ones presented in figure 15a for the stability region of the ferromagnetic phase at the same values of $U, n$ and $t_{3}$, one can see that there is an obvious correlation between the shape of the noninteracting DOS and ferromagnetism. Indeed, the ferromagnetic state is stabilized only for these values of frustration parameters $t_{2}$, $t_{3}$, which lead to the single peaked noninterating DOS at the band edge. From the moment that two or more peaks appear in the noninteracting DOS at the band edge (by changing $t_{2}$ or $t_{3}$ ), ferromagnetism is suppressed.

\subsubsection{Long-range hopping}

With respect to the above presented results, it is natural to ask what happens in the situation when also the next-nearest neighbor hopping terms will be included (for example, the $t_{4}$ and $t_{5}$ terms are of the same order as the $t_{3}$ term). To answer this question, we performed the same calculations with the same one-parametric formula [equation [2.1p] as was used in the section 2.1. To reveal the possible stability regions of the ferromagnetic state in the generalized Hubbard model on the SSL, let us first examine the effects of the long-range hopping on the behavior of the non-interacting DOS. As mentioned above, just this quantity, particularly, the appearance of the single-peaked DOS near the band edge could be used as a good indicator for the emergence of ferromagnetism in the interacting systems. The noninteracting DOS of the $U=0$ Hubbard model on the SSL of size $L=200 \times 200$, obtained by exact diagonalization of $H$ (for $U=0$ ) is shown in figure 17 for several different values ot the long-range-hopping parameter 

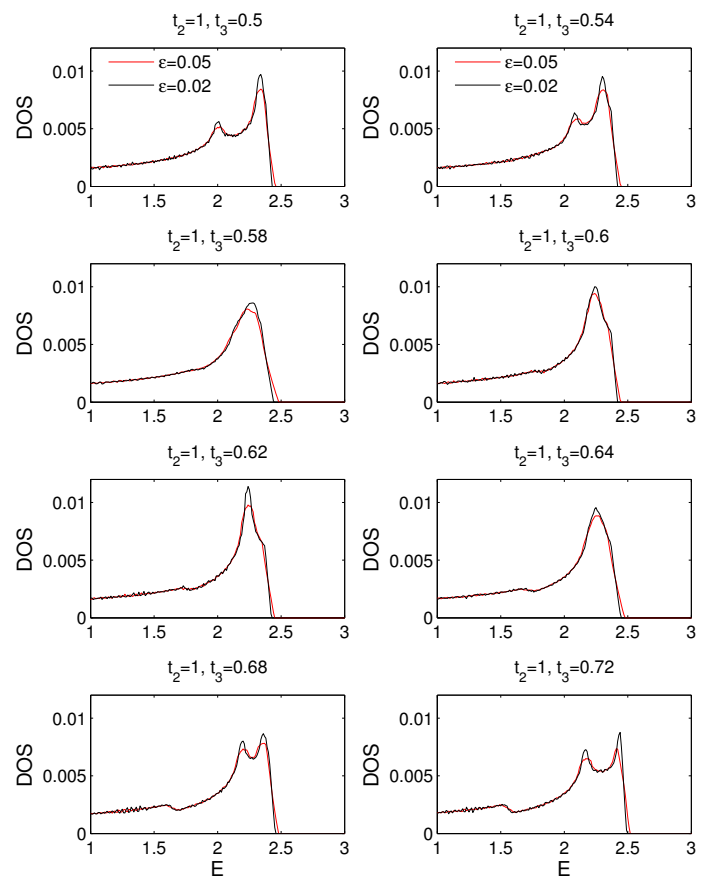

Figure 16. (Colour online) Non-interacting DOS calculated numerically for $t_{2}=1$ and different values of $t_{3}$ (near $t_{3}=0.6$ ) on the finite cluster of $L=200 \times 200$ sites [36].

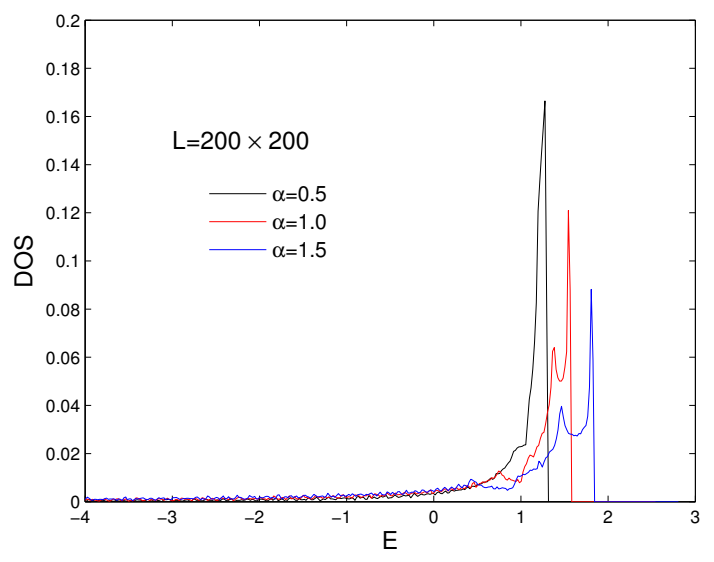

Figure 17. (Colour online) Non-interacting DOS calculated numerically for different values of the longrange hopping parameter $\alpha$ on the finite cluster of $L=200 \times 200$ sites [37].

$\alpha=\ln q$. One can see that the DOS is strongly asymmetric with practically all spectral weight located at the upper band edge, which in accordance with some previous works indicates a possible region of ferromagnetism for electron concentrations above the half-filed band case $n>1$. Moreover, the DOS is double-peaked for $\alpha \geqslant 1$ and single-peaked for $\alpha<1$, indicating ferromagnetism in the limit of small values of $\alpha$.

To verify these conjectures, we performed exhaustive numerical studies of the model for a wide range 

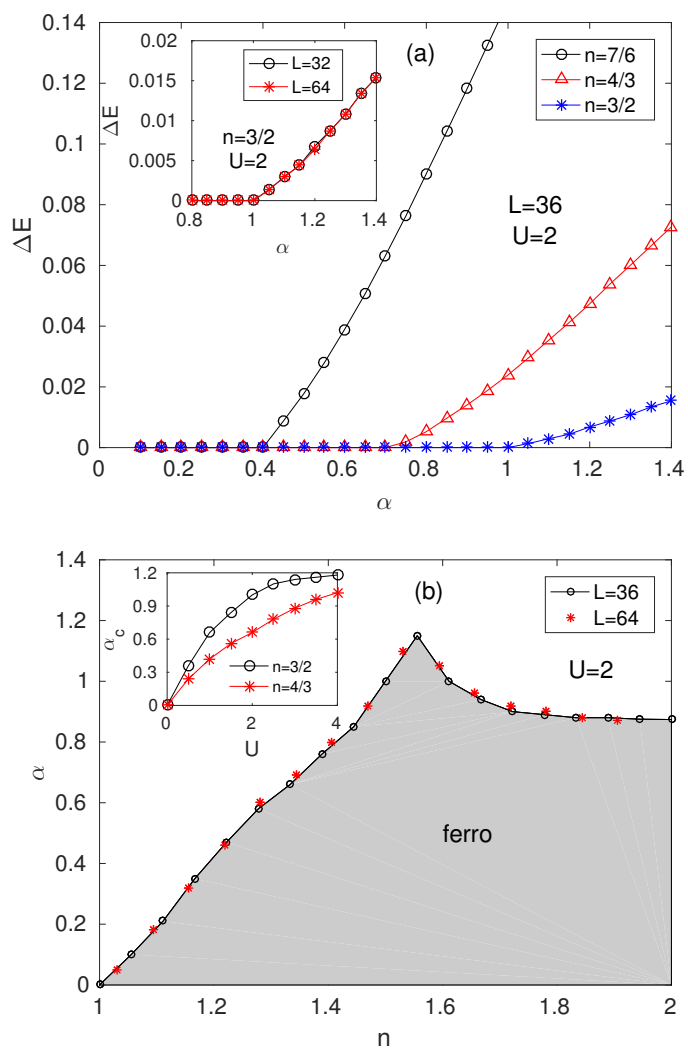

Figure 18. (Colour online) (a) The difference $\Delta E=E_{f}-E_{\min }$ between the ferromagnetic state $E_{f}$ and the lowest ground-state energy from $E_{g}\left(S_{z}\right)$ as a function of the long-range hopping parameter $\alpha$ calculated for three different electron concentrations on the finite cluster of $L=6 \times 6$ sites $(U=2)$. The inset shows $\Delta E$ calculated for $n=3 / 2$ on clusters of $L=6 \times 6$ and $L=8 \times 8$ sites. (b) The ground-state phase diagram of the generalized Hubbard model with long-range hopping on the SSL calculated for two different finite clusters of $L=36$ and $L=64$ sites at $U=2$. The inset shows the critical value of the long-range hopping parameter $\alpha_{c}$ (bellow which the ground state is ferromagnetic) as a function of the Coulomb interaction $U$ calculated for two different electron concentrations $n=3 / 2$ and $n=4 / 3$ at $U=2$ and $L=36[37]$.

of the model parameters $U, \alpha$ for all even electron concentrations above the half-filled band case. The nature of the ground state of the Hubbard model on the SSL is identified by the projector quantum Monte Carlo method [30] with $\theta \sim 30$ and a time slice of $\Delta \theta=0.05$ which suffices to reach well converged values of the observables. Typical results of our PQMC calculations obtained on the finite cluster of $L=6 \times 6$ sites for three different electron concentrations $(n=7 / 6,4 / 3$ and $n=3 / 2)$ are shown in figure 18 . It is seen that for all electron concentrations, there exists a finite interval of $\alpha$ values where $\Delta E=0$ indicating the fact that ferromagnetism in the generalized Hubbard model with exponentially decaying hopping amplitudes is not restricted only to high electron concentrations, as in the case of the Hubbard model on the SSL with the first, second and third nearest-neighbor electron hopping, but also extends to smaller values of electron concentrations. To exclude the finite-size effect, we also performed the same calculations on the larger cluster of $L=8 \times 8$ sites. One can see that the finite-size effects on the stability region of the ferromagnetic phase are negligible and thus these results can be satisfactorily extrapolated to the thermodynamic limit $L=\rightarrow \infty$.

The second step of our study was to specify more precisely the stability region of the ferromagnetic state. For this reason, we performed exhaustive numerical studies of the model for a wide range of model parameters $U, \alpha$ and $n$. The results obtained are presented in the form of the ground-state phase diagram 
Table 1. The hopping amplitudes $t_{i}^{\prime}$ for the first, second, third, forth and fifth nearest neighbors on the real Archimedean lattice. Note that on the real Archimedean lattice corresponding to rare-earth Shastry-Sutherland magnets $t_{1}^{\prime}=t_{1}=t_{2}$ [37].

\begin{tabular}{|c|c|c|c|c|c|}
\hline \hline$\alpha$ & $t_{1}^{\prime}$ & $t_{2}^{\prime}$ & $t_{3}^{\prime}$ & $t_{4}^{\prime}$ & $t_{5}^{\prime}$ \\
\hline 0.1 & 1 & 0.9594 & 0.9294 & 0.9110 & 0.8699 \\
0.2 & 1 & 0.9205 & 0.8638 & 0.8299 & 0.7567 \\
0.3 & 1 & 0.8831 & 0.8028 & 0.7561 & 0.6582 \\
0.4 & 1 & 0.8473 & 0.7461 & 0.6888 & 0.5725 \\
0.5 & 1 & 0.8129 & 0.6935 & 0.6276 & 0.4980 \\
0.6 & 1 & 0.7799 & 0.6445 & 0.5717 & 0.4332 \\
0.7 & 1 & 0.7483 & 0.5990 & 0.5208 & 0.3768 \\
0.8 & 1 & 0.7179 & 0.5567 & 0.4745 & 0.3278 \\
0.9 & 1 & 0.6888 & 0.5174 & 0.4323 & 0.2851 \\
1.0 & 1 & 0.6609 & 0.4809 & 0.3938 & 0.2480 \\
\hline \hline
\end{tabular}

in the $n-\alpha$ plane (see figure 18p). These results clearly support our aforementioned conjecture that ferromagnetic state in the Hubbard model with the exponentially decaying hopping amplitudes on the SSL is robust and exists for all electron concentrations above the half-filled band case $n>1$. The width of the ferromagnetic domain very strongly depends on the values of electron concentrations and reaches its maximum for intermediate values of $n$. Again, we performed the evaluation of the finite size effects by calculating the same phase diagram on the larger $8 \times 8$ cluster, but no significant effects were observed and thus these results can be also satisfactorily extrapolated to the thermodynamic limit.

We also performed the same calculations for different values of the Coulomb interaction in order to demonstrate the interplay between the long-range electron hopping and the on-site electron interaction. The results of our numerical calculations obtained for two different electron concentrations $(n=4 / 3$ and $n=3 / 2$ ) are summarized in the inset to figure $18 \mathrm{p}$ and they clearly demonstrate strong effects of the Coulomb interaction that considerably shifts $\alpha_{c}$ to higher values. This leads to a very important conclusion, namely, that correlation effects can, under some conditions (e.g., a special lattice structure), stabilize the ferromagnetic state in strongly correlated systems.

To reveal the physical limits of the model, in table 1 we presented the actual values of the first, second, third, fourth and fifth nearest-neighbour hopping amplitudes on the real Archimedean lattice, corresponding to the real structure of rare-earth tetraborides, for selected values of the model parameter $\alpha$. One can see that the smallest values of $\alpha(\alpha \leqslant 0.2)$ are of interest only from the academic point of view (a very small decay of hopping amplitudes $t_{i}^{\prime}$ ) and of physical interest are only those values of $\alpha$, which are greater than 0.2, representing the realistic situation in the Shastry-Sutherland materials. The ferromagnetic domain in this limit is still robust indicating a significant impact of lattice structure and long-range hopping on the stabilization of ferromagnetism in the strongly correlated electron systems.

\section{Conclusion}

In this review we presented the results of our numerical calculations concerning the problem of stabilization of ferromagnetism in the generalized Hubbard model, considered herein as a generic model for a description of itinerant ferromagnetism in narrow-band systems. In particular, we examined the effects of (i) the long-range hopping, (ii) the correlated hopping, (iii) the long-range Coulomb interaction, (iv) the flat bands and (v) the lattice structure. We found that each of the above mentioned terms plays a significant role in stabilizing the ferromagnetic state, and for each of these terms we determined the domains in the parametric space of the model where the the ferromagnetic state is the ground state of the generalized Hubbard model. Our results can be summarized as follows: (i) It is found that the longrange hopping with exponentially decaying hopping amplitudes stabilizes the ferromagnetic state for a wide range of electron interactions $U$ and electron concentrations $n$ for both the one-dimensional and 
two-dimensional case. In the one dimensional case, the ferromagnetic state is stable (above some critical value of Coulomb interaction) for all electron concentrations $n>1$, while in the two-dimensional case, it is stable only for electron concentrations from the interval $1<n<n_{c}<2$, where the critical value of electron concentration $n_{c}$ strongly depends on the strength of Coulomb interaction $U$. (ii) Examining the combined effects of long-range and correlated hopping, we found that ferromagnetic state for nonzero $q, U$ and $n$ is further stabilized with an increasing strength of the correlated hopping term $t^{\prime}$. The effect is especially strong for intermediate and strong values of $q$. There even exists some critical value of $q$ above which the ground state is ferromagnetic for all nonzero $U$. With an increasing $t^{\prime}$, this critical value shifts to lower values of $q$ (that represent a much more realistic type of electron hopping) and the ferromagnetic domain correspondingly increases. (iii) Similarly, examining the combined effects of the long-rage hopping and long-range Coulomb interaction (both considered with exponentially decaying amplitudes), we found that the long-range interaction plays a crucial role in the stabilization of the ferromagnetic state for electron concentrations $n \leqslant 1$, while the long-range hopping for $n>1$. (iv) With respect to the influence of flat bands on the formation and stabilization of the ferromagnetic state within the Hubbard model, we found that at fixed $U$, the ferromagnetic state is stabilized with increasing concentration of holes $(1-n)$ in the system, and at a fixed $n$, the ferromagnetic state is generally stabilized with an increasing $U$. (v) The study of the Hubbard model on the SSL with the first, second and third couplings showed that there are strong correlations between ferromagnetism and the shape of the noninteracting density of states (the lattice structure). In particular, it is found that ferromagnetism is stabilized only for these values of frustration parameters $\left(t_{1}, t_{2}, t_{3}\right)$, which lead to the single peaked noninterating density of states at the band edge. From the moment that two or more peaks appear in the noninteracting density of states at the band edge, the ferromagnetic state is suppressed. (vi) In addition, we found that the ferromagnetic domain for the case of the first, second and third nearest neighbors is considerably enhanced, when long-range hopping with exponentially decaying amplitudes is considered. All these results point to the fact that the absence of ferromagnetism in the ordinary Hubbard model with the nearest-neighbour hopping and on-site Coulomb interaction is obviously the consequence of oversimplified description of electron hopping and electron interactions on the lattice.

\section{Acknowledgements}

This work was supported by the Slovak Research and Development Agency under the contracts no. APVV-20-0293, APVV-17-0020, the Slovak Grant Agency Vega under the contract No. 2-0112-18 and the projects ITMS 26230120002, ITMS 26210120002 (Slovak infrastructure for high-performance computing) supported by the Research and Development Operational Programme funded by the ERDF.

\section{References}

1. Hubbard J., Proc. R. Soc. London A, 1963, 276, 238, doi $10.1098 /$ rspa.1963.0204.

2. Nagaoka Y., Phys. Rev., 1966, 147, 392, doi:10.1103/PhysRev.147.392

3. Müller-Hartmann E., J. Low. Temp. Phys., 1995, 99, 342.

4. Lieb E. H., Phys. Rev. Lett., 1989, 62, 1201, doi 10.1103/PhysRevLett.62.1201

5. Ulmke M., Eur. Phys. J. B, 1998, 1, 301, doi 10.1007/s100510050186

6. Pandey S., Singh A., Phys. Rev. B, 2007, 75, 064412, doi 10.1103/PhysRevB.75.064412.

7. Pieri P., Mod. Phys. Lett. B, 1996, 10, 1277, doi:10.1142/S0217984996001449.

8. Salerno M., Z. Phys. B, 1996, 99, 469, doi:10.1007/s002570050064

9. Salerno M., Z. Phys. B, 1996, 101, 619, doi:10.1007/s002570050254

10. Mielke A., J. Phys. A, 1992, 25, 4335, doi:10.1088/0305-4470/25/16/011

11. Tasaki H., Phys. Rev. Lett., 1992, 69, 1608, doi:10.1103/PhysRevLett.69.1608.

12. Mielke A., Tasaki H., Commun. Math. Phys., 1993, 158, 341, doi 10.1007/BF02108079.

13. Tasaki H., Progr. Theor. Phys., 1998, 99, 489, doi:10.1143/PTP.99.489

14. Katsura H., Maruyama I., Tanaka A., Tasaki H., EPL, 2010, 91, 57007, doi $10.1209 / 0295-5075 / 91 / 57007$

15. Khodel V. A., Shaginyan V. R., JETP Lett., 1990, 51, 553.

16. Volovik G. E., JETP Lett., 1991, 53, 222. 
17. Gofron K., Campuzano J. C., Abrikosov A. A., Lindroos M., Bansil A., Ding H., Koelling D., Dabrowski B., Phys. Rev. Lett., 1994, 73, 3302, doi 10.1103/PhysRevLett.73.3302

18. Tasaki H., Phys. Rev. Lett., 1994, 73, 1158, doi:10.1103/PhysRevLett.73.1158.

19. Mielke A., Phys. Rev. Lett., 1999, 82, 4312, doi:10.1103/PhysRevLett.82.4312

20. Tanaka A., Ueda H., Phys. Rev. Lett., 2003, 90, 067204, doi $10.1103 /$ PhysRevLett.90.067204

21. Maksymenko M., Honecker A., Moessner R., Richter J., Derzhko O., Phys. Rev. Lett., 2012, 109, 096404, doi:10.1103/PhysRevLett.109.096404

22. Gulacsi Z., Kampf A., Vollhardt D., Phys. Rev. Lett., 2010, 105, 266403, doi 10.1103/PhysRevLett.105.266403

23. Volovik G. E., Phys.-Usp., 2018, 61, No. 1, 89-98, doi 10.3367/ufne.2017.01.038218.

24. Dagotto E., Rev. Mod. Phys., 1994, 66, 763, doi:10.1103/RevModPhys.66.763

25. Peschel I., Wang X., Kaulke M., Hallberg K. (Eds.), Density-Matrix Renormalization. Lecture Notes in Physics 528, Springer, Berlin, 1999.

26. Schollwock U., Rev. Mod. Phys., 2005, 77, 259, doi 10.1103/RevModPhys.77.259

27. Hallberg K. A., Adv. Phys., 2006, 55, 477, doi $10.1080 / 00018730600766432$

28. Sorella S., Baroni S., Car R., Parinello M., Europhys. Lett., 1989, 8, 663, doi:10.1209/0295-5075/8/7/014

29. Loh E. Y., Gubernatis J. E., In: Modern Problems of Condensed Matter Physics, Hanke W., Kopaev Y. (Eds.), North Holland, Amsterdam, 1992.

30. Imada M., Quantum Monte Carlo Methods in Condensed Matter Physics, World Scientific, Singapore, 1993.

31. Farkašovský P., Phys. Rev. B, 2002, 66, 012404, doi:10.1103/PhysRevB.66.012404.

32. Farkašovský P., Čencariková H., Cent. Eur. J. Phys., 2013, 11, 119, doi 10.2478/s11534-012-0135-7

33. Farkašovský P., EPL, 2015, 110, 47007, doi 10.1209/0295-5075/110/47007.

34. Farkašovský P., Czech. J. Phys., 2004, 54, 419, doi:10.1007/s10582-004-0110-7.

35. Farkašovský P., Eur. Phys. J. B, 2012, 85, 253, doi 10.1140/epjb/e2012-30306-9

36. Farkašovský P., Eur. Phys. J. B, 2018, 91, 74, doi 10.1140/epjb/e2018-80650-7

37. Farkašovský P., J. Supercond. Nov. Magn., 2019, 32, 1007-1011, doi:10.1007/s10948-018-4788-3

38. Farkašovský P., J. Phys.: Condens. Matter, 1995, 7, 3001, doi 10.1088/0953-8984/7/15/007

39. Farkašovský P., J. Phys.: Condens. Matter, 1995, 7, 9775, doi $10.1088 / 0953-8984 / 7 / 50 / 012$

40. Shvaika A. M., Phys. Rev. B, 2003, 67, 075101, doi:10.1103/PhysRevB.67.075101

41. Shvaika A. M., Condens. Matter Phys., 2014, 17, 43704, doi:10.5488/CMP.17.43704

42. Čenčariková H., Farkašovský P., Condens. Matter Phys., 2011, 14, 42701, doi:10.5488/CMP.14.42701

43. Farkašovský P., Hudáková N., J. Phys.: Condens. Matter, 2001 14, 499, doi $10.1088 / 0953-8984 / 14 / 3 / 318$.

44. Hirsch J. E., Physica C, 1989, 158, 326, doi 10.1016/0921-4534(89)90225-6.

45. Amadon J. C., Hirsch J. E., Phys. Rev. B, 1996, 54, 6364, doi:10.1103/PhysRevB.54.6364

46. Kollar M., Vollhardt D., Phys. Rev. B, 2001, 63, 045107, doi 10.1103/PhysRevB.63.045107.

47. Farkašovský P., Phys. Rev. B, 1998, 57, 14722, doi 10.1103/PhysRevB.57.14722.

48. Lyzwa R., Domanski Z., Phys. Rev. B, 1994, 50, 11381, doi $: 10.1103 /$ PhysRevB.50.11381.

49. Domanski Z., Lyzwa R., Erdos P., J. Magn. Magn. Mater., 1995, 140, 1205, doi:10.1016/0304-8853(94)00652-0

50. Fath G., Domanski Z., Lemanski R., Phys. Rev. B, 1995, 52, 13910, doi 10.1103/PhysRevB.52.13910

51. Falicov L. M., Kimball J. C., Phys. Rev. Lett., 1969, 22, 997, doi 10.1103/PhysRevLett.22.997.

52. Gu S. J., Fan R., Lin H. Q., Phys. Rev. B, 2008, 76, 125107, doi:10.1103/PhysRevB.76.125107.

53. Farkašovský P., Phys. Rev. B, 2008, 77, 085110, doi:10.1103/PhysRevB.77.085110

54. Ueltschi D., J. Stat. Phys., 2004, 116, 681, doi:10.1023/B:JOSS.0000037231.88815.04

55. Shastry B. S., Sutherland B., Physica B+C, 1981, 108, 1069, doi:10.1016/0378-4363(81)90838-X.

56. Kageyama H., Yoshimura K., Stern R., Mushnikov N. V., Onizuka K., Kato M., Kosuge K., Slichter C. P., Goto T., Ueda Y., Phys. Rev. Lett., 1999, 82, 3168, doi 10.1103/PhysRevLett.82.3168.

57. Kodama K., Takigawa M., Horvatic M., Berthier C., Kageyama H., Ueda Y., Miyahara S., Becca F., Mila F., Science, 2002, 298, 395, doi 10.1126/science.1075045

58. Liu H. D., Lin H. F., Tao H. S., Liu W. M., Sci. Rep., 2014, 4, 4829, doi 10.1038/srep04829 


\section{Зонний феромагнетизм у вузькозонних металах}

\section{П. Фаркашовськи}

Інститут експериментальної фізики, Словацька академія наук вул. Ватсонова 47, 04353 Кошіце, Словаччина

3 моменту запровадження у 1963 р., модель Хаббарда стала однією з найбільш популярних в літературі, що використовуються для вивчення колективних явищ у вузькозонних металах (феромагнетизм, переходи "метал-діелектрик", хвилі зарядової густини, високотемпературна надпровідність). Серед усіх цих колективних явищ проблема зонного феромагнетизму в моделі Хаббарда має найдовшу історію. Незважаючи на вражаючу дослідницьку активність у минулому, розуміння фізиками мікроскопічних механізмів, що призводять до стабілізації зонного феромагнетизму в моделі Хаббарда (вузькозонні метали), поки що $\epsilon$ далеко не повним. У цьому огляді представлено наші числові результати з цієї проблеми, отримані точною діагоналізацією для малих кластерів, методом ренормгрупи для матриці густини та квантовим методом Монте-Карло в рамках різних узагальнень моделі Хаббарда. Особливу увагу приділено опису вирішальних механізмів (взаємодій), що сприяють стабілізації феромагнітного стану, а саме: (i) дальніх перескоків, (ii) скорельованих перескоків, (iii) далекосяжної кулонівської взаємодії, (iv) плоских зон, (v) структури ґратки. Хоча більшість представлених результатів отримано для одновимірного випадку, але також обговорюється і вплив збільшення розмірності системи на їі феромагнітний стан.

Ключові слова: зонний феромагнетизм, системи скорельованих електронів, модель Габбарда 


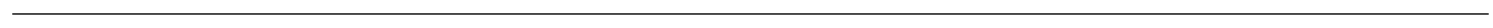

